[15] G. Herzog, On linear operators having supercyclic vectors, Studia Math. 103 (1992), 295-298.

[16] G. Herzog and R. Lemmert, Über Endomorphismen mit dichten Bahnen, Math. Z. 213 (1993), 473-477.

[17] H. Heuser, Funktionalanalysis, 2nd ed., Teubner, 1986.

[18] M. Mbekhta et A. Ouahab, Perturbations des opérateurs s-réguliers et continuité de certains sous-espaces dans le domaine quasi-Fredholm, Publ. IRMA Lille 24 (1991), No. 10

[19] M. Ó. Searcóid and T. T. West, Continuity of the generalized kernel and range of semi-Fredholm operators, Math. Proc. Cambridge Philos. Soc. 105 (1989), 513-522.

[20] S. Rolewicz, On orbits of elements, Studia Math. 32 (1969), 17-22.

[21] W. Rudin, Functional Analysis, McGraw-Hill, 1973.

$[22]$ H. Salas, A hypercyclic operator whose adjoint is also hypercyclic, Proc. Amer. Math. Soc. 112 (1991), 765-770.

[23] C. Schmoeger, Ascent, descent and the Atkinson region in Banach algebras, II, Ricerche Mat, to appear.

MATHEMATISCHES INSTITUT I

UNIVERSITÄT KARLSRUHE

POSTFACH 6980

D-76128 KARLSRUHE, GERMANY

\section{On quasi-multipliers}

by

ZIYA ARG UN (Ankara) and K. ROWLANDS (Aberystwyth)

Abstract. A quasi-multipliers is a generalization of the notion of a left (right, double) multiplier. The first systematic account of the general theory of quasi-multipliers on a Banach algebra with a bounded approximate identity was given in a paper by McKennon in 1977. Further developments have been made in more recent papers by Vasudevan and Goel, Kassem and Rowlands, and Lin. In this paper we consider the quasi-multipliers of algebras not hitherto considered in the literature. In particular, we study the quasimuitipliers of $A^{\text {t"}}$-algebras, of the algebra of compact operators on a Banach space, and of the Pedersen ideal of a $C^{*}$-algebra. We also consider the strict topology on the quasimultiplier space $Q M(A)$ of a Banach algebra, $A$ with a bounded approximate identity. We multiplier space $Q M(A)$ of a Banach algebra. $A$ with a bounded approximate identity. We
prove that, if $M_{l}(A)$ (resp. $M_{r}(A)$ ) denotes the algebra of left (right) multipliers on $A$, then $M_{l}(A)+M_{r}(A)$ is strictly dense in $Q M(A)$, thereby generalizing a theorem due to Lin.

1. Introduction. A quasi-multiplier is a generalization of the notion of a left (right, double) multiplier, and was first introduced by Akemann and Pedersen in $([1], \S 4)$. The first systematic account of the general theory of quasi-multipliers on a Banach algebra with a bounded approximate identity was given in a paper by McKennon [14] in 1977. Further developments have been made as a result of more recent contributions by Vasudevan and Goel [21], [22], Kassem and Rowlands [11], and Lin [13]. In this paper we study the quasi-multipliers of algebras not hitherto considered in the literature.

We begin by outlining the necessary background results on quasi-multipliers and then proceed to consider the quasi-multipliers $Q M(A)$ of an $A^{*}$. algebra $A$ in particular, we improve a result due to Vasudevan and Goel ([21], Theorem 3.4) on extending a quasi-multiplier from an $A^{*}$-algebra to its auxiliary norm completion. The result enables us to define an "auxiliary" norm on $Q M(A)$ and, in the special case when $Q M(A)$ is a Banach algebra, we prove that under certain conditions $Q M(A)$ is an $A^{*}$-algebra. In the literature topologies other than the norm topology have been defined on the quasi-multiplier space and properties established for the resulting locally

1991 Mathematics Subject Classification: Primary $46 \mathrm{H} 05$. 
convex spaces (see, for example, [11] and [131). In $\$ 3$ we consider the strict topology on the quasi-multiplier space of a Banach algebra $A$ with a bounded approximate identity. In particular, we prove that, if $M_{l}(A)$ (resp. $M_{r}(A)$ ) denotes the algebra of left (right) multipliers on $A$, then $M_{l}(A)+M_{r}(A)$ is strictly dense in $Q M(A)$, thereby generalizing a theorem due to Lin ([13], Theorem 9.3).

In $\S 4, A$ is the algebra of approximable operators on a Banach space $X$ (that is, operators that can be approximated, in the operator norm, by operators of finite rank), and our investigations lead to characterizations for $Q M(A)$ and $Q M\left(A^{* *}\right)$. For example, if $X^{*}$ has the bounded approximation property then $Q M(A)$ is topologically isomorphic to $M_{r}(A)$ and, if in addition $X^{*}$ has the Radon-Nikodym property, then $Q M\left(A^{* * *}\right)$ is topologically isomorphic to $M_{l}\left(A^{* *}\right)$. In the final section we study the quasi-multipliers of the Pedersen ideal $K_{A}$ of a $C^{*}$-algebra $A$. In this case the quasi-multipliers are not necessarily continuous. Nevertheless, the space $\delta\left(K_{A}\right)$ of quasi-multipliers on $K_{A}$, with the quasi-strict topology $\gamma$, has a number of interesting properties. In particular, we show that $\delta\left(K_{A}\right)$ is $\gamma$-complete, and, for certain $C^{*}$-algebras, $K_{A}$ is $\gamma$-dense in $\delta\left(K_{A}\right)$. We also establish a characterization for the dual space $\left(\delta\left(K_{A}\right), \gamma\right)^{*}$.

2. Preliminaries and quasi-multipliers of $A^{*}$-algebras. Let $A$ be a Banach algebra. A mapping $m: A \times A \rightarrow A$ is said to be a quasi-multiplier on $A$ if

$$
m(a b, c)=a m(b, c) \text { and } m(a, b c)=m(a, b) c
$$

for all $a, b, c \in A$. Let $Q M(A)$ denote the set of all bilinear jointly continuous quasi-multipliers on $A$. If $A$ is a Banach algebra with a bounded two-sided approximate identity (abbreviated to a.i. in the sequel), then every quasimultiplier belongs to $Q M(A)$ ([14], Theorem 1$)$, and $Q M(A)$ is a Banach space with respect to the norm

$$
\|m\|=\sup \{\|m(a, b)\|: a, b \in A,\|a\| \leq 1,\|b\| \leq 1\}
$$

([14], Theorem 2). If the products $a \circ m$ and $m \circ a$ are defined by

$$
(a \circ m)(x, y)=m(x a, y), \quad(m \circ a)(x, y)=m(x, a y)
$$

$(m \in Q M(A), x, y, a \in A)$, then $Q M(A)$ becomes a Banach $A$-module.

A mapping $T: A \rightarrow A$ is called a left (resp. right) multiplier on $A$ if $T(a b)=(T a) b(\operatorname{resp} . T(a b)=a(T b))$ for all $a, b$ in $A$, and $T^{+}$is called a multiplier if it is both a left and right multiplier on $A$. Let $M_{0}(A)$ (resp. $M_{l}(A), M_{r}(A)$ ) be the set of all continuous linear (left, right) multipliers on $A$. Then both $M_{l}(A)$ and $M_{r}(A)$ are closed subalgebras of the Bar nach algebra $\mathcal{L}(A)$ of all continuous linear operators on $A$ and $M_{0}(A)$ is a closed commutative subalgebra of $\mathcal{L}(A)$. A pair $(S, T)$ of mappings
$S, T: A \rightarrow A$ is said to be a double multiplier on $A$ if $a S b=(T a) b$ for all $a, b \in A$. If $M(A)$ denotes the set of all continuous linear double multipliers on $A$, then, for each $(S, T) \in M(A)$, we have $S \in M_{l}(A)$, $T \in M_{r}(A)$, and $\|(S, T)\|=\max (\|S\|,\|T\|)$ defines a norm on $M(A)$ relative to which it is a Banach algebra. For further details on the algebras of left, riglat and clouble multipliers on a Banach algebra we refer the reader to ([20], $§ 3)$.

Each of the linear mappings

$$
\begin{gathered}
\Phi: A \rightarrow Q M(A), \quad \lambda: M_{l}(A) \rightarrow Q M(A), \\
\varrho: M_{r}(A) \rightarrow Q M(A), \quad \Psi: M(A) \rightarrow Q M(A),
\end{gathered}
$$

defined respectively by

$$
\begin{gathered}
(\Phi(a))(x, y)=x a y, \quad(\lambda(S))(x, y)=x S y, \\
(\varrho(T))(x, y)=(T x) y, \quad \Psi(S, T)=\lambda(S),
\end{gathered}
$$

is a norm decreasing embedding; if, in addition, $A$ has a minimal a.i. $\left\{e_{\alpha}\right.$ : $\alpha \in I\}$ (that is, $\left\|e_{x x}\right\| \leq 1$ for all $\alpha \in I$ ), then the mappings are isometric.

A bounded a.i. $\left\{e_{\kappa}: \alpha \in I\right\}$ in $A$ is said to be an ultra-approximate identity if, for all $m \in Q M(A)$ and $a \in A$, the nets $\left\{m\left(a, e_{\alpha}\right)\right\}$ and $\left\{m\left(e_{\alpha}, a\right)\right\}$ are Cauchy ([14], p. 110). In this case $\lambda$ and $\varrho$ are surjective; for, if $m \in Q M(A)$, the mappings $S, T$ on $A$ defined by

$$
S a=\lim _{\alpha} m\left(e_{\alpha}, a\right), \quad T a=\lim _{\alpha} m\left(a, e_{\alpha}\right),
$$

belong to $M_{l}(A)$ and $M_{r}(A)$ respectively, and $\lambda(S)=m=\varrho(T)$. Under these circumstances we can use either of the isomorphisms $\lambda$ or $\varrho$ to define multiplication in $Q M(A)$. Thus, for example, the equation

$$
\left(m_{1} \odot m_{2}\right)(a, b)=m_{1}\left(a, \lim _{\alpha} m_{2}\left(e_{\alpha}, b\right)\right)
$$

defines a product in $Q M(A)$, and, if we assume that the ultra-approximate identity is minimal, then $Q M(A)$ becomes a Banach algebra, with $\lambda$ (resp. Q) an isometric algebraic isomorphism of $M_{l}(A)\left(M_{r}(A)\right)$ onto $Q M(A)$.

A bilinear mapping $m: A \times A \rightarrow A$ can be extended in two natural ways to a bilinear map $A^{* *} \times A^{* * *} \rightarrow A^{* *}$; we outline the construction in stages, as follows:

$$
\text { (i) } \begin{array}{rlrl}
m^{*}: A^{*} \times A \rightarrow A^{*}, & \left\langle b, m^{*}(f, a)\right\rangle & =\langle m(a, b), f\rangle, \\
m^{* *}: A^{* *} \times A^{*} \rightarrow A^{*}, & \left\langle a, m^{* *}(F, f)\right\rangle & =\left\langle m^{*}(f, a), F\right\rangle, \\
m^{* * *}: A^{* *} \times A^{* *} \rightarrow A^{* *}, & \left\langle f, m^{* * *}(F, G)\right\rangle & =\left\langle m^{* *}(G, f), F\right\rangle ; \\
& \left(m^{\prime}\right)^{*}: A \times A^{*} \rightarrow A^{*}, & \left\langle b,\left(m^{\prime}\right)^{*}(a, f)\right\rangle & =\langle m(b, a), f\rangle, \\
\left(m^{\prime}\right)^{* * *}: A^{*} \times A^{* *} \rightarrow A^{*}, & \left\langle a,\left(m^{\prime}\right)^{* *}(f, F)\right\rangle & =\left\langle\left(m^{\prime}\right)^{*}(a, f), F\right\rangle, \\
\left(m^{\prime}\right)^{* * *}: A^{* *} \times A^{* * *} \rightarrow A^{* *}, & \left\langle f\left(m^{\prime}\right)^{* * *}(F, G)\right\rangle & =\left\langle\left(m^{\prime}\right)^{* *}(f, F), G\right\rangle
\end{array}
$$


$\left(a, b \in A, f \in A^{*}, F, G \in A^{* *}\right)$. It is easy to check that when $m$ is continuous then $m^{*}, m^{* *}, m^{* * *},\left(m^{\prime}\right)^{*},\left(m^{\prime}\right)^{* *},\left(m^{\prime}\right)^{* * *}$ are continuous. Moreover, by routine calculations we can show that, if $m \in Q M(A)$, then $m^{* * *}$ and $\left(m^{\prime}\right)^{* * *}$ are quasi-multipliers on $\left(A^{* *}, \cdot\right)$ and $\left(A^{* *}, *\right)$ respectively, where . (resp.*) denotes the first (second) Arens product on $A^{* *}$. (For the defi. nitions of the Arens products on $A^{* *}$, we refer the reader to $([20], \S 4)$; in particular, we note that $\left(A^{* *}, \cdot\right)$ (resp. $L\left(A^{* *}, *\right)$ ) has a right (left) identity if and only if $A$ has a bounded right (left) approximate identity ([3], p. 146, Proposition 9)). It is also straightforward to show that $m^{* * *}(\widehat{a}, \widehat{b})=m(\widehat{a}, b)$ for all $a, b \in A$.

The following is a simpler proof of ([21], Theorem 2.1).

THEOREM 2.1. Let $A$ be a Banach algebra and suppose that $E$ (resp. I) is a right (left) identity in $A^{* *}$ with respect to the first (second) Arens product. Then the mapping $m \rightarrow m^{* * *}(E, I)$ is a topological linear isomorphism of $Q M(A)$ into $A^{* *}$, with

$$
\|m\| \leq\left\|m^{* * *}(E, I)\right\| \leq\|m\|\|E\|\|I\| .
$$

Proof. It is clear that the mapping $m \rightarrow m^{* *}(E, I)$ is linear. For any $a, b \in A$,

$$
\|m(a, b)\|=\|\widehat{m(a, b)}\|=\left\|m^{* * *}(\widehat{a}, \widehat{b})\right\|,
$$

and since $m^{* * *}$ is a quasi-multiplier on $A^{* *}$ we have

$$
\|m(a, b)\|=\left\|a \cdot m^{* * *}(E, I) \cdot b\right\| \leq\left\|m^{* * *}(E, I)\right\|\|a\|\|b\|,
$$

which implies that

$$
\|m\| \leq\left\|m^{* * *}(E, I)\right\|
$$

The right side inequality follows from the fact that $m^{* * *} \in Q M\left(A^{* *}, \cdot\right)$ and that $\left\|m^{* * *}\right\| \leq\|m\|$.

COROLlary 2.2. If $\|E\|=\|I\|=1$, then the mapping $m \rightarrow m^{* * *}(E, I)$ is isometric.

Similarly we can show that $m \rightarrow m^{* * *}(I, E)$ is a topological linear isomorphism of $Q M(A)$ into $A^{* *}$. The following generalizes a result due to Vasudevan and Goel ([22], Corollary 3.1).

THeOREM 2.3. Let $A$ be a Banach algebra. The image of $\Phi(A)$ under the mapping $m \rightarrow m^{* * *}(I, E)$ is $\widehat{A}$.

Proof. We show that, for each $a \in A,(\Phi(a))^{* * *}(I, E)=\widehat{a}$. Since $E$ (resp. $I)$ is a right (left) identity in $\left(A^{* *},\right)$ ) (resp. $\left.\left(A^{* *}, *\right)\right), E$ (resp. $\left.I\right)$ is the weak*-limit of a bounded right (left) a.i. in $A$. Thus, for any $f$ in $A^{*}$,

$$
\begin{aligned}
\left\langle f,(\Phi(a))^{* * *}(I, E)\right\rangle & =\left\langle(\Phi(a))^{* *}(E, f), I\right\rangle=\lim _{\beta}\left\langle\widetilde{e}_{\beta},(\Phi(a))^{* *}(E, f)\right\rangle \\
& =\lim _{\beta}\left\langle(\Phi(a))^{*}\left(f, \widetilde{e}_{\beta}\right), E\right\rangle=\lim _{\alpha} \lim _{\beta}\left\langle e_{\alpha},(\Phi(a))^{*}\left(f, \widetilde{e}_{\beta}\right)\right\rangle \\
& =\lim _{\alpha} \lim _{\beta}\left\langle\widetilde{e}_{\beta} a e_{\alpha}, f\right\rangle=\langle a, f\rangle,
\end{aligned}
$$

which implies that $(\Phi(a))^{* * *}(I, E)=\widehat{a}$, as required.

Let $A_{\sigma r}^{* *}=\left\{F \in A^{* *}: a \cdot F \cdot b \in A\right.$ for all $\left.a, b \in A\right\}$. It is easy to show that $A_{\sigma}^{* *}$ is a closed subspace of $A^{* *}$ and the equation

$$
\sigma(\widehat{F)(a}, b)=a \cdot F \cdot b \quad\left(a, b \in A, F \in A_{\sigma}^{* *}\right)
$$

defines a norm decreasing linear mapping $\sigma$ of $A_{\sigma}^{* *}$ into $Q M(A)$. The kernel of $\sigma$ is $\left(A A^{*} A\right)^{\perp}$. For the sake of completeness we give the following properties of $\sigma$.

LeMma 2.4 (cf. [21], Theorem 2.4). Let $A$ be a Banach algebra and suppose that $E$ (resp. I) is a right (left) identity in $A^{* *}$ with respect to the first (second) Arens product. Then

(i) $F \in A_{\sigma}^{* *}$ if and only if there exist an $m$ in $Q M(A)$ and $a G \in \operatorname{ker} \sigma$ such that $F=m^{* * * *}(E, I)+G$, and

(ii) $\sigma$ maps $A_{\sigma}^{* *}$ onto $Q M(A)$.

Proof. (i) Suppose that $F \in A_{\sigma}^{* *}$. Then the mapping $(\widehat{a}, \widehat{b}) \rightarrow a \cdot F \cdot b$ defines an element of $Q M(\widehat{A})$ and so, since $Q M(A)$ and $Q M(\widehat{A})$ are isomorphic (via the correspondence $\left.m \rightarrow m^{* * *}\right|_{\widehat{A}}$ ), there exists an $m \in Q M(A)$ such that $m^{* * *}(\widehat{a}, \widehat{b})=a \cdot F \cdot b$. Thus, for any $f \in A^{*}$,

$$
\left\langle f, a \cdot m^{* * *}(E, I) \cdot b\right\rangle=\left\langle f, m^{* * *}(\widehat{a}, \widehat{b})\right\rangle=\langle f, a \cdot F \cdot b\rangle,
$$

which implies that $a \cdot\left(m^{* * *}(E, I)-F\right) \cdot b=0$; that is, $m^{* * *}(E, I)-F \in \operatorname{ker} \sigma$, and so $F=m^{* * * *}(E, I)+G$ for some $G \in$ ker $\sigma$, as required.

On the other hand, suppose that $F \in A^{* *}$ has the representation $F=$ $m^{* * * *}(E, I)+G$ for some $G \in \operatorname{ker} \sigma$ and $m \in Q M(A)$. Then

$$
a \cdot F \cdot b=m^{* * *}(\widehat{a}, \widehat{b})+a \cdot G \cdot b=m^{* * *}(\widehat{a}, \widehat{b})
$$

since $G \in$ ker $\sigma$; that is, $F \in A_{\sigma}^{* *}$

(ii) Let $m$ be any element of $Q M(A)$. Then $m^{* * *}(E, I) \in A_{\sigma}^{* *}$ and it is easy to show that $\sigma\left(m^{* * *}(E, I)\right)=m$; that is, $\sigma$ is a surjection.

We now turn our attention to Banach *-algebras. If $A$ is a Banach *-algebra, then we can define an involution in $Q M(A)$ by setting

$$
m^{*}(a, b)=\left(m\left(b^{*}, a^{*}\right)\right)^{*}
$$

It is clear that, if $a \rightarrow a^{*}$ is continuous in $A$, then $m \rightarrow m^{*}$ is a continuous mapping in $Q M(A)$. 
THEOREM 2.5. Let $A$ be a Banach *-algebra and suppose that $E$ (resp. $I$ ) is a right (left) identity for the first (second) Arens product on $A^{* *}$. If, in addition, $\left(A A^{*} A\right)^{\perp}=\{0\}$, then $m \rightarrow m^{* * *}(E, I)$ is a continuous linear *-isomorphism of $Q M(A)$ into $A^{* *}$.

Proof. In view of Theorem 2.1 it is enough to prove that $m^{*} \rightarrow$ $\left(m^{* * *}(E, I)\right)^{*}$. (In this proof we are using the $*$-notation in different senses but it should not give rise to confusion.) First it is easy to show that the identities $\left\langle a, f^{*}\right\rangle=\overline{\left\langle a^{*}, f\right\rangle},\left\langle f, F^{*}\right\rangle=\overline{\left\langle f^{*}, F\right\rangle}\left(a \in A, f \in A^{*}, F \in A^{* *}\right)$ define involutions in $A^{*}$ and $A^{* *}$ respectively. A straightforward application of the above identities enables us to prove the following:

$$
(b * f \cdot a)^{*}=a^{*} * f^{*} \cdot b^{*}, \quad a \cdot F^{*} \cdot b=\left(b^{*} \cdot F \cdot a^{*}\right)^{*} .
$$

Thus, since $m^{*}$ is a quasi-multiplier on $A,\left(m^{*}\right)^{* * *}$ is a quasi-multiplier on $A^{* *}$, and so

$$
\begin{aligned}
a \cdot\left(m^{*}\right)^{* * *}(E, I) \cdot b & =\left(m^{*}\right)^{* * *}(\widehat{a}, \widehat{b})=m^{*}(a, b) \\
& =\left(m\left(\widehat{b^{*}, a^{*}}\right)\right)^{*}=\left(m^{* * *}\left(\widehat{b}^{*}, \widehat{a^{*}}\right)\right)^{*} \\
& =\left(b^{*} \cdot m^{* * *}(E, I) \cdot a^{*}\right)^{*}=a \cdot\left(m^{* * *}(E, I)\right)^{*} \cdot b .
\end{aligned}
$$

It follows that $\left(m^{*}\right)^{* * *}(E, I)-\left(m^{* * *}(E, I)\right)^{*} \in\left(A A^{*} A\right)^{\perp}$, and so we have $\left(m^{*}\right)^{* * *}(E, I)=\left(m^{* * *}(E, I)\right)^{*}$, as required.

The following is an improvement of a result given by Vasudevan and Goel in ([21], Theorem 3.4).

THEOREM 2.6. Let $A$ be an $A^{*}$-algebra with a bounded a.i. and let $\mathcal{U}$ be its auxiliary norm completion. Then, for each $m \in Q M(A)$, there exists a unique $\bar{m}$ in $Q M(\mathcal{U})$ such that $m=\left.\bar{m}\right|_{A \times A}$.

Proof. Let $|\cdot|$ denote the auxiliary norm in $A$. By ([19], Corollary 4.1.16) there exists a positive number $\beta$ such that $|a| \leq \beta\|a\|$ for all $a \in A$. It follows that, for each $g \in \mathcal{U}^{*}$, the restriction of $g$ to $A, g_{A}$ say, is an element of $(A,\|\cdot\|)^{*}$ (abbreviated to $A^{*}$ in the sequel), with $\left\|g_{A}\right\| \leq \beta|g|$ Thus, if $F \in A^{* *}$, the functional $\bar{F}$ on $\mathcal{U}^{*}$ defined by $\langle g, \bar{F}\rangle=\left\langle g_{A}, F\right\rangle$ is an element of $\mathcal{U}^{* *}$.

Let $m$ be any element of $Q M(A)$ and let $G=m^{* * *}(E, E)$, where $E$ is a right identity for the first Arens product and a left identity for the second. Now $G \in A_{\sigma}^{* *}$. We show that $\bar{G} \in \mathcal{U}_{\sigma *}^{* *}$, where $\mathcal{U}_{\sigma}^{* *}$ is clefined in an analogous way to $A_{\sigma}^{* *}$. First we prove that, for $a, b \in A$,

$$
\pi_{\mathcal{U}}(\sigma(G)(a, b))=\pi_{\mathcal{U}}(a) \cdot \bar{G} \cdot \pi_{\mathcal{U}}(b),
$$

where $\pi_{\mathcal{U}}$ denotes the canonical embedding of $\mathcal{U}$ in $\mathcal{U}^{* *}$. For any $g \in \mathcal{U}^{*}$,

$$
\begin{aligned}
\left\langle g, \pi_{\mathcal{U}}(\sigma(G)(a, b))\right\rangle & =\langle\sigma(G)(a, b), g\rangle=\left\langle\sigma(G)(a, b), g_{A}\right\rangle \\
& =\left\langle g_{A}, a \cdot G \cdot b\right\rangle=\left\langle b * g_{A} \cdot a, G\right\rangle .
\end{aligned}
$$

On the other hand,

$$
\left\langle g, \pi_{\mathcal{U}}(a) \cdot \bar{G} \cdot \pi_{\mathcal{U}}(b)\right\rangle=\left\langle(b * g \cdot a)_{A}, G\right\rangle,
$$

and since $(b * g \cdot a)_{A}=b * g_{A} \cdot a$, we have

$$
\left\langle g, \pi_{\mathcal{U}}(a) \cdot \bar{G} \cdot \pi_{\mathcal{U}}(b)\right\rangle=\left\langle b * g_{A} \cdot a, G\right\rangle .
$$

Thux (2.2) follows from (2.3) and (2.4).

Let $u, v \in \mathcal{U}$. Since $A$ is $|\cdot|$-dense in $\mathcal{U}$, there exist sequences $\left\{a_{n}\right\},\left\{b_{m}\right\}$ in $A$ such that $a_{n} \rightarrow u, b_{m} \rightarrow v$, with respect to the auxiliary norm on $\mathcal{U}$. Thus

$$
\lim _{n, m} \pi_{\mathcal{U}}\left(a_{n}\right) \cdot \bar{G} \cdot \pi_{\mathcal{U}}\left(b_{m}\right)=\pi_{\mathcal{U}}(u) \cdot \bar{G} \cdot \pi_{\mathcal{U}}(v),
$$

and so by (2.1),

$$
\pi_{\mathcal{U}}(u) \cdot \bar{G} \cdot \pi_{\mathcal{U}}(v)=\lim _{n, m} \pi_{\mathcal{U}}\left(\sigma(G)\left(a_{n}, b_{m}\right)\right)
$$

It follows that $\pi_{\mathcal{U}}(u) \cdot \bar{G} \cdot \pi_{\mathcal{U}}(v) \in \pi_{\mathcal{U}}(\mathcal{U})$; that is, $\bar{G} \in \mathcal{U}_{\sigma}^{* *}$, as required. Let $\bar{m}=\sigma(\bar{G})$. Then $\bar{m} \in Q M(\mathcal{U})$, and, for $a, b \in A$,

$$
\begin{aligned}
\pi_{\mathcal{U}}(\bar{m}(a, b)) & =\pi_{\mathcal{U}}(a) \cdot \bar{G} \cdot \pi_{\mathcal{U}}(b) \\
& =\pi_{\mathcal{U}}(\sigma(G)(a, b))=\pi_{\mathcal{U}}(m(a, b)),
\end{aligned}
$$

which implies that $\left.\bar{m}\right|_{\mathcal{A} \times A}=m$. The uniqueness of $m$ follows immediately from the fact that $A$ is auxiliary norm dense in $\mathcal{U}$.

The above result enables us to make the following.

DEFINITION 2.7, For each $m \in Q M(A)$, we define the "auxiliary" norm on $Q M(A)$ by

$$
|m|=|\bar{m}|
$$

our use of the terminology will be justified later.

Before we make a further study of the space $(Q M(A), \mid \cdot 1)$ we require some results on the double multipliers of an $A^{*}$-algebra; the theorems proved are variants of ([12], 'Theorems 3.3-3.7).

THeOrum 2.8. Let $A$ be an $A^{*}$-algebra with a bounded a.i. and let $\mathcal{U}$ be its auxiliary norm completion. Then each $(S, T) \in M(A)$ has a unique caterision to a double multiplier $\left(S^{\prime}, T^{\prime}\right) \in M(\mathcal{U})$.

Proof. We first show that each $S \in M_{l}(A)$ has a unique extension to an element $S^{\prime} \in M_{l}(\mathcal{U})$. With $E$ as in the proof of Theorem 2.6, let $F=S^{* *} E$. For $g \in \mathcal{U}^{*}$, the equation $\langle g, \bar{F}\rangle=\left\langle g_{A}, F\right\rangle$, where $g_{A}$ denotes the restriction of $g$ to $A$, defines an element $\vec{F} \in U^{* *}$. Moreover, $F \cdot a \in \pi u(A)(a \in A)$; for, $\bar{F} \cdot a=\bar{F} \cdot a$ and since $F \cdot a=\overline{S a}$, it follows that $\bar{F} \cdot a \in \pi_{\mathcal{U}}(A)$. Since 
$\mathcal{U}$ is the $|\cdot|$-closure of $A$ the above implies that $\bar{F} \cdot u \in \pi_{\mathcal{U}}(\mathcal{U})$ for all $u \in \mathcal{U}$. This enables us to define a mapping $S^{\prime}: \mathcal{U} \rightarrow \mathcal{U}$ by

$$
\pi_{\mathcal{U}}\left(S^{\prime} u\right)=\bar{F} \cdot \pi_{\mathcal{U}}(u) \quad(u \in \mathcal{U}) .
$$

It is straightforward to show that $S^{\prime} \in M_{l}(\mathcal{U})$. Moreover, $S^{\prime}$ is an extension of $S$; for, if $a \in A$, then

$$
\pi_{\mathcal{U}}\left(S^{\prime} a\right)=\bar{F} \cdot \pi_{\mathcal{U}}(a)=\overline{F \cdot a}=\widehat{S a}=\pi_{\mathcal{U}}(S a),
$$

which implies that $S^{\prime} a=S a$; that is, $\left.S^{\prime}\right|_{A}=S$. The extension is unique since $A$ is $|\cdot|$-dense in $\mathcal{U}$

In a similar way we can prove that each $T \in M_{r}(A)$ has a unique extension to an element $T^{\prime} \in M_{r}(\mathcal{U})$. The equation corresponding to (2.4) is given by

$$
\pi_{\mathcal{U}}\left(T^{\prime} u\right)=\pi_{\mathcal{U}}(u) \cdot \bar{F}
$$

It. follows that, if $(S, T) \in M(A)$, then $\left(S^{\prime}, T^{\prime}\right)$ is its unique extension to $M(\mathcal{U})$.

THEOREM 2.9. Let $A$ be an $A^{*}$-algebra with a bounded a.i. Then $M(A)$ is an $A^{*}$-algebra.

Proof. We recall that $M(A)$ is a Banach algebra with respect to the norm $\|(S, T)\|=\max (\|S\|,\|T\|)$. In addition, since $A$ is a *-algebra, $M(A)$ is a Banach *-algebra, the involution being defined by $(S, T)^{*}=\left(T^{*}, S^{*}\right)$, where $S^{*} a=\left(S a^{*}\right)^{*}$ and $T^{*} a=\left(T a^{*}\right)^{*}(a \in A)$. Thus it is enough to show that we can define an auxiliary norm on $M(A)$.

By Theorem 2.8, each $(S, T) \in M(A)$ has a unique extension to an element $\left(S^{\prime}, T^{\prime}\right) \in M(\mathcal{U})$. Define the auxiliary norm on $M(A)$ by $|(S, T)|=$ $\left|\left(S^{\prime}, T^{\prime}\right)\right|$. It is easy to check that $\left(S T^{*}\right)^{\prime}=S^{\prime}\left(T^{\prime}\right)^{*}$ and $\left(S^{*} T\right)^{\prime}=\left(S^{\prime}\right)^{*} T^{\prime}$ and so

$$
\begin{aligned}
\left|(S, T)(S, T)^{*}\right| & =\left|\left(\left(S T^{*}\right)^{\prime},\left(S^{*} T\right)^{\prime}\right)\right|=\left|\left(S^{\prime}\left(T^{\prime}\right)^{*},\left(S^{\prime}\right)^{*} T^{\prime}\right)\right| \\
& =\left|\left(S^{\prime}, T^{\prime}\right)\left(S^{\prime}, T^{\prime}\right)^{*}\right|=\left|\left(S^{\prime}, T^{\prime}\right)\right|^{2}
\end{aligned}
$$

since $M(\mathcal{U})$ is a $B^{*}$-algebra. Thus $\left|(S, T)(S, T)^{*}\right|=|(S, T)|^{2}$; that is, $M(A)$ with the norms $\|(\cdot, \cdot)\|$ and $|(\cdot, \cdot)|$ is an $A^{*}$-algebra.

By using the same arguments as the ones used to prove ([12], Theorem 3.5) (resp. ([12], Theorem 3.7)) we can show that $M(A)$ is algebraically *isomorphic and auxiliary norm isometric to the subalgebra $K=\{(V, W) \in$ $M(\mathcal{U}): V(A) \subseteq A, W(A) \subseteq A\}$ of $M(\mathcal{U})$ (resp. to a subalgebra of $\left(\mathcal{U}^{* *}, \cdot\right)$ ).

THEOREM 2.10. Let $A$ be an $A^{*}$-algebra with a bounded $a . i$. and let $\mathcal{U}$ be its auxiliary norm completion. Then $Q M(A)$ is linearly *-isomorphic and auxiliary norm isometric to a subspace of $Q M(\mathcal{U})$.
Proof. Let $\mathcal{P}=\{m \in Q M(\mathcal{U}): m(A \times A) \subseteq A\}$. Clearly $\mathcal{P}$ is a *-subspace of $Q M(\mathcal{U})$. If $m \in \mathcal{P}$, then $\left.m\right|_{A \times A}$ is a quasi-multiplier on $A$, and so, since $A$ has a bounded a.i., $\left.m\right|_{A \times A} \in Q M(A)$. Let $\widetilde{\Psi}$ denote the mapping of $Q M(A)$ into $\mathcal{P}$ defined by $\widetilde{\Psi}(q)=\bar{q}$, where $\bar{q}$ is the unique extension of $q \in Q M(A)$ as given in Theorem 2.6. It follows that $\widetilde{\Psi}$ is a linear surjection of $Q M(A)$ onto $\mathcal{P}$. It is routine to show that, for $m \in Q M(A)$, $\left.\widetilde{\Psi}\left(m^{*}\right)\right|_{A \times A}=\left(\left.\widetilde{\Psi}(m)\right|_{A \times A}\right)^{*}$, which implies that $\widetilde{\Psi}\left(m^{*}\right)=(\widetilde{\Psi}(m))^{*}$; that is, $\tilde{\Psi}$ is a $*$-isomorphism. Finally, it is clear from Definition 2.7 that $\widetilde{\Psi}$ is auxiliary norm isometric.

If $A$ has a minimal ultra-approximate identity, then $Q M(A)$ is a Banach algebra. In this case the above theorem may be strengthened to give the following.

THEOREM 2.11. Let $A$ be an $A^{*}$-algebra with a minimal ultra-approximate identity. Then $Q M(A)$ is algebraically *-isomorphic and auxiliary norm isometric to a subalgebra of $Q M(\mathcal{U})$.

Proof. Let $\left\{e_{\alpha}\right\}$ denote the minimal ultra-approximate identity in $A$. For $m_{1}, m_{2} \in Q M(A)$, the product $m_{1} \odot m_{2}$ is given by

$$
\left(m_{1} \odot m_{2}\right)(a, b)=m_{1}\left(a, \lim _{\alpha} m_{2}\left(e_{\alpha}, b\right)\right) \text {. }
$$

We now extend the above definition to define a product in $\mathcal{P}$.

Since $A$ is an $A^{*}$-algebra there exists a positive number $\beta$. such that $|a| \leq \beta\|a\|$ for all $a \in A$. Moreover, since $A$ is auxiliary norm dense in $\mathcal{U}$, it follows that, for $m \in Q M(A)$ and $u \in \mathcal{U}$, the nets $\left\{\bar{m}\left(e_{\alpha}, u\right)\right\}$ and $\left\{\bar{m}\left(u, e_{\alpha}\right)\right\}$ are Cauchy in $\mathcal{U}$ and hence convergent. Thus we can define a product in $\mathcal{P}$ by setting

$$
\left(\overline{m_{1}} \odot \overline{m_{2}}\right)(u, v)=\overline{m_{1}}\left(u, \lim _{\alpha} \overline{m_{2}}\left(e_{\alpha}, v\right)\right)
$$

so that $\mathcal{P}$ is a subalgebra of $Q M(\mathcal{U})$. We also note that $\left.\overline{m_{1}} \odot \overline{m_{2}}\right|_{A \times A}=$ $m_{1} \odot m_{2}$, and so

$$
\overline{m_{1}} \odot \overline{m_{2}}=\overline{m_{1} \odot m_{2}} \text {. }
$$

It follows from the above and Theorem 2.10 that $\widetilde{\Psi}$ is an algebraic *-isomorphism of $Q M(A)$ onto $\mathcal{P}$.

With $A$ as in Theorem 2.11, the mappings $\lambda, \varrho$ and $\Psi$ are isometric embeddings of $\left(M_{l}(A),\|\cdot\|\right),\left(M_{r}(A),\|\cdot\|\right)$ and $(M(A),\|\cdot\|)$ respectively into $(Q M(A),\|\cdot\|)$. If, instead, we consider the auxiliary norms on $M(A)$ and $Q M(A)$, then we have the following

THEOREM 2.12. Let $A$ be an $A^{*}$-algebra with a minimal ultra-approximate identity. Then there exists a positive number $\beta$ such that

(i) $\beta^{-1}|(S, T)| \leq|\Psi(S, T)| \leq|(S, T)|$ for all $(S, T) \in M(A)$, 
(ii) $\beta^{-1}|S| \leq|\lambda(S)| \leq|S|\left(S \in M_{l}(A)\right)$

(iii) $\beta^{-1}|T| \leq|\varrho(T)| \leq|T|\left(T \in M_{r}(A)\right)$.

Proof. (i) Let $\left\{e_{\alpha}\right\}$ denote the minimal ultra-approximate identity in $A$. Since $A$ is an $A^{*}$-algebra there exists a positive number $\beta$ such that $|a| \leq$ $\beta\|a\|$ for all $a \in A$. If $(S, T) \in M(A)$ and $\left(S^{\prime}, T^{\prime}\right)$ its unique extension to $M(\mathcal{U})$, then, for any $u \in \mathcal{U}$,

$$
\left|S^{\prime} u\right|=\lim _{\alpha}\left|e_{\alpha} S^{\prime} u\right|=\lim _{\alpha}\left|\Psi\left(S^{\prime}, T^{\prime}\right)\left(e_{\alpha}, u\right)\right| \leq \beta\left|\Psi\left(S^{\prime}, T^{\prime}\right)\right||u|,
$$

which implies that $\left|S^{\prime}\right| \leq \beta\left|\Psi\left(S^{\prime}, T^{\prime}\right)\right|$. (We are using the same notation to denote the embeddings of $M(A)$ in $Q M(A)$ and $M(\mathcal{U})$ in $Q M(\mathcal{U})$ but this should not cause any confusion.) Since $\left.\left(\Psi\left(S^{\prime}, T^{\prime}\right)\right)\right|_{A \times A}=\Psi(S, T)$, we have $|\Psi(S, T)|=\left|\Psi\left(S^{\prime}, T^{\prime}\right)\right|$. Now $|(S, T)|=\left|\left(S^{\prime}, T^{\prime}\right)\right|=\left|S^{\prime}\right|$, and so it follows that $\beta^{-1}|(S, T)| \leq|\Psi(S, T)|$. The right hand side inequality holds since $\Psi:(M(A),|\cdot|) \rightarrow(Q M(A),|\cdot|)$ is norm decreasing.

(ii) and (iii) may be proved by the same methods.

THeOREM 2.13. Let $A$ be as in Theorem 2.12. Then, for any $m \in$ $Q M(A)$

$$
\beta^{-1}|m|^{2} \leq\left|m \odot m^{*}\right| \leq \beta^{2}|m|^{2} .
$$

Proof. Let $m \in Q M(A)$. Since $\Psi$ is surjective there exists an $(S, T) \in$ $M(A)$ such that $m=\Psi(S, T)$. We also note that $m^{*}=\Psi\left((S, T)^{*}\right)$. Thus

$$
\begin{aligned}
\left|m \odot m^{*}\right| & =\left|\Psi(S, T) \odot \Psi\left((S, T)^{*}\right)\right|=\left|\Psi\left((S, T)(S, T)^{*}\right)\right| \\
& \leq\left|(S, T)(S, T)^{*}\right|=|(S, T)|^{2}
\end{aligned}
$$

since $M(A)$ is an $A^{*}$-algebra by Theorem 2.9. By Theorem 2.12(i),

$$
\left|m \odot m^{*}\right| \leq \beta^{2}|m|^{2} ;
$$

also

$$
\begin{aligned}
|m|^{2} & =|\Psi(S, T)|^{2} \leq|(S, T)|^{2}=\left|(S, T)(S, T)^{*}\right| \\
& \leq \beta\left|\Psi(S, T) \odot \Psi\left((S, T)^{*}\right)\right|=\beta\left|m \odot m^{*}\right|
\end{aligned}
$$

and so

$$
\beta^{-1}|m|^{2} \leq\left|m \odot m^{*}\right| \leq \beta^{2}|m|^{2},
$$

as required.

COROLlaRY 2.14. If $\beta=1$, then $|\cdot|$ satisfies the $B^{*}$-condition and $Q M(A)$ is an $A^{*}$-algebra.

The above corollary justifies the use of the term "auxiliary" norm in Definition 2.7
3. Quasi-multipliers and the strict topology. Let $A$ be a Banach algebra with a bounded a.i.

DEFINITION 3.1. The left strict $\beta_{l}$, right strict $\beta_{r}$, strict $\beta$ and quasi-strict $\gamma$ topologies on $Q M(A)$ are defined respectively by the following families of semi-norms:

$$
\begin{aligned}
\text { (i) } m \rightarrow\|a \circ m\|, \\
\text { (ii) } m \rightarrow\|m \circ a\|, \\
\text { (iii) } m \rightarrow\|a \circ m\| \text { and } m \rightarrow\|m \circ a\|, \\
\text { (iv) } m \rightarrow\|a \circ m \circ b\|
\end{aligned}
$$

$(a, b \in A, m \in Q M(A))$.

Clearly $\gamma \subseteq \beta_{l}$ and $\beta_{r} \subseteq \beta$. The properties of $(Q M(A), \gamma)$ have been studied in some detail in $([11], \S 3)$; in this section we turn our attention to $(Q M(A), \beta)$.

THEOREM 3.2. $Q M(A)$ is $\beta$-complete.

Proof. We first note that, for each $a \in A$ and $m \in Q M(A)$, the mappings $S_{a}$ and $T_{a}$, given by

$$
S_{a}(b)=m(a, b), \quad T_{a}(b)=m(b, a),
$$

define elements in $M_{l}(A)$ and $M_{r}(A)$ respectively, and it is easy to show that $\lambda\left(S_{a b}\right)=a \circ m$ and $\varrho\left(T_{a}\right)=m \circ a$.

Let $\left\{m_{\alpha}: \alpha \in I\right\}$ be a $\beta$-Cauchy net in $Q M(A)$ and let $a \in A$. It follows from the definition of the $\beta$-topology that the nets $\left\{\lambda\left(S_{a}\right)_{\alpha}\right\}$ and $\left\{\varrho\left(T_{a}\right)_{\alpha}\right\}$, where $\left(S_{a}\right)_{\alpha} b=m_{\alpha}(a, b)$ and $\left(T_{a}\right)_{\alpha} b=m_{\alpha}(b, a)$, are norm-Cauchy in $Q M(A)$. Since $\lambda$ and $\varrho$ are topological embeddings, the nets $\left\{\left(S_{a}\right)_{\alpha}\right\}$ and $\left\{\left(T_{a}\right)_{\alpha}\right\}$ are norm-Cauchy in $M_{l}(A)$ and $M_{r}(A)$ respectively. Both $M_{l}(A)$ and $M_{r}(A)$ are Banach spaces and so there exist $S^{(a)}$ in $M_{l}(A)$ and $T^{(a)}$ in $M_{r}(A)$ such that

$$
\left\|\left(S_{a}\right)_{\alpha}-S^{(a)}\right\| \rightarrow 0, \quad\left\|\left(T_{a}\right)_{\alpha}-T^{(a)}\right\| \rightarrow 0 .
$$

Since $\gamma \subseteq \beta$, the net $\left\{m_{\alpha}\right\}$ is $\gamma$-Cauchy. The space $Q M(A)$ is $\gamma$-complete ([14], Theorem 6) and so there exists an element $m_{0}$ in $Q M(A)$ such that

$$
\lim _{\alpha} m_{\alpha}(x, y)=m_{0}(x, y)
$$

for all $x, y \in A$. For any $b, c \in A$,

$$
\begin{aligned}
\left(\lambda\left(S^{(a)}\right)\right)(b, c) & =\lim _{\alpha}\left(\lambda\left(\left(S_{a}\right)_{\alpha}\right)\right)(b, c)=\lim _{\alpha} b m_{\alpha}(a, c) \\
& =\left(a \circ m_{0}\right)(b, c)
\end{aligned}
$$

which implies that $\lambda\left(S^{(a)}\right)=a \circ m_{0}$. Similarly we can prove that $\varrho\left(T^{(a)}\right)=$ $m_{0} \circ a$. Thus

$$
\left\|a \circ m_{\alpha}-a \circ m_{0}\right\|=\left\|\lambda\left(S_{a}\right)_{\alpha}-\lambda\left(S^{(a)}\right)\right\| \leq\left\|\left(S_{a}\right)_{\alpha}-S^{(a)}\right\| \rightarrow 0
$$


and

$\left\|m_{\alpha} \circ a-m_{0} \circ a\right\|=\left\|\varrho\left(T_{a}\right)_{\alpha}-\varrho\left(T^{(a)}\right)\right\| \leq\left\|\left(T_{a}\right)_{\alpha}-T^{(a)}\right\| \rightarrow 0$,

which implies that $m_{0}$ is the $\beta$-limit of the net $\left\{m_{\alpha}\right\}$; that is, $Q M(A)$ is $\beta$-complete, as required.

Since $\gamma \subseteq \beta$, every $\beta$-bounded set is $\gamma$-bounded. But the $\gamma$-bounded and norm bounded subsets of $Q M(A)$ coincide ([11], Theorem 3.2), and so every $\beta$-bounded subset of $Q M(A)$ is norm bounded. Clearly every norm bounded subset of $Q M(A)$ is $\beta$-bounded. We thus have the following

THEOREM 3.3. $(Q M(A), \beta),(Q M(A), \gamma)$ and $(Q M(A),\|\cdot\|)$ have the same bounded sets.

Our next aim is to generalize a theorem due to Lin ([13], Theorem 9.3). Let

$$
\begin{aligned}
& A_{\mu}^{* *}=\left\{F \in A^{* *}: F \cdot a \in A \text { for all } a \in A\right\}, \\
& A_{\nu}^{* *}=\left\{F \in A^{* *}: a \cdot F \in A \text { for all } a \in A\right\}, \\
& A_{\sigma}^{* *}=\left\{F \in A^{* *}: a \cdot F \cdot b \in A \text { for all } a, b \in A\right\} .
\end{aligned}
$$

The strict topology on $A^{* *}$ is defined to be the locally convex topology determined by the semi-norms $F \rightarrow\|F \cdot a\|$ and $F \rightarrow\|a \cdot F\|\left(a \in A, F \in A^{* *}\right)$.

It is clear that $A_{\mu}^{* *}+A_{\nu}^{* *} \subseteq A_{\sigma}^{* *}$, but, in fact, more is true:

LEMMA 3.4. $A_{\mu}^{* *}+A_{\nu}^{* *}$ is strictly dense in $A_{\sigma}^{* *}$.

Proof. Let $\left\{e_{\alpha}: \alpha \in I\right\}$ be a bounded a.i. for $A$, with $\left\|e_{\alpha}\right\| \leq C(\alpha \in I)$, and suppose that $F \in A_{\sigma}^{* *}$. For each $\alpha \in I$, let $F_{\alpha}=e_{\alpha} \cdot F-e_{\alpha} \cdot \bar{F} \cdot e_{\alpha}+F \cdot e_{\alpha}$. Clearly $F \cdot e_{\alpha} \in A_{\nu}^{* *}$ and $e_{\alpha} \cdot F \in A_{\mu}^{* *}$, and so to complete the proof we show that $F_{\alpha}$ converges strictly to $F$. Let $a \in A$. Then

$$
\begin{aligned}
\left\|a \cdot F_{\alpha}-a \cdot F\right\| & \leq\left\|a \cdot F \cdot e_{\alpha}-a e_{\alpha} \cdot F \cdot e_{\alpha}\right\|+\left\|a e_{\alpha} \cdot F-a \cdot F\right\| \\
& \leq\left\|a-a e_{\alpha}\right\|\|F\| C+\left\|a e_{\alpha}-a\right\|\|F\| \rightarrow 0
\end{aligned}
$$

similarly we can show that $\left\|F_{\alpha} \cdot a-F \cdot a\right\| \rightarrow 0$.

THEOREM 3.5. Let $A$ be a Banach algebra with a bounded a.i. Then $M_{l}(A)+M_{r}(A)$ is strictly dense in $Q M(A)$.

Proof. Let $m \in Q M(A)$ and suppose that $\left\{e_{\alpha}: \alpha \in I\right\}$ is a bounded a.i. for $A$, with $\left\|e_{\alpha}\right\| \leq C(\alpha \in I)$. For each $\alpha \in I$, define mappings $S_{\alpha}$ and $T_{\alpha}$ by

$$
S_{\alpha}(a)=m\left(e_{\alpha}, a-e_{\alpha} a\right), \quad T_{\alpha}(a)=m\left(a, e_{\alpha}\right) \quad(a \in A) .
$$

Clearly $S_{\alpha} \in M_{l}(A)$ and $T_{\alpha} \in M_{r}(A)$.

Let $F=m^{* * *}(E, E)$, where $E$ is a weak*-cluster point of $\left\{\widehat{e}_{\alpha}\right\}$. For each $\alpha \in I$ we prove the following.

(i) $\left(\varrho\left(T_{\alpha}\right)\right)^{* * *}(E, E)=F \cdot e_{\alpha}$, (ii) $\left(\left(\lambda\left(S_{\alpha}\right)\right)^{\prime}\right)^{* * *}(E, E)=e_{\alpha} \cdot F-e_{\alpha} \cdot F \cdot e_{\alpha}$.

For (i), $\left\langle f,\left(\varrho\left(T_{\alpha}\right)\right)^{* * *}(E, E)\right\rangle=\left\langle\left(\varrho\left(T_{\alpha}\right)\right)^{* *}(E, f), E\right\rangle\left(f \in A^{*}\right)$; routine calculations show that $\left(\varrho\left(T_{\alpha}\right)\right)^{* *}(E, f)=T_{\alpha}^{*}(E \cdot f)$, and so, since $f$ is arbitrary in $A^{*}$, it follows that

Also, for any $f$ in $A^{*}$,

$$
\left(\varrho\left(T_{\alpha}\right)\right)^{* * *}(E, E)=T_{\alpha}^{* *} E
$$

$$
\begin{aligned}
\left\langle f, T_{\alpha}^{* * *} E\right\rangle & =\left\langle T_{\alpha}^{*} f, E\right\rangle=\lim _{\beta}\left\langle e_{\beta}, T_{\alpha}^{*} f\right\rangle=\lim _{\beta}\left\langle m\left(e_{\beta}, e_{\alpha}\right), f\right\rangle \\
& =\lim _{\beta}\left\langle e_{\beta}, m^{* *}\left(\widehat{e}_{\alpha}, f\right)\right\rangle=\left\langle f, m^{* * *}\left(E, \widehat{e}_{\alpha}\right)\right\rangle \\
& =\left\langle f, m^{* * *}\left(E, E * e_{\alpha}\right)\right\rangle=\left\langle f, m^{* * *}(E, E) \cdot e_{\alpha}\right\rangle,
\end{aligned}
$$

which implies that

$$
T_{\alpha}^{* *} E=m^{* * *}(E, E) \cdot e_{\alpha}
$$

(i) follows from (3.1) and (3.2)

For (ii), we first note that, if $S \in M_{l}(A)$ then $S^{* *} \in M_{l}\left(A^{* *}, *\right)$. For each $S \in M_{l}(A)$, let $\tilde{\lambda}\left(S^{* *}\right)$ be the element of $Q M\left(A^{* *}, *\right)$ defined by

$$
\left(\tilde{\lambda}\left(S^{* *}\right)\right)(F, G)=F * S^{* *} G \text {. }
$$

A routine calculation shows that

$$
\left((\lambda(S))^{\prime}\right)^{* * *}(F, G)=\left(\widetilde{\lambda}\left(S^{* *}\right)\right)(F, G),
$$

so that, in particular, for each $\alpha \in I$,

$$
\left(\left(\lambda\left(S_{\alpha}\right)\right)^{\prime}\right)^{* * *}(E, E)=E * S_{\alpha}^{* *} E=S_{\alpha}^{* *} E
$$

For each $\alpha \in I$ and $f \in A^{*}$,

$$
\begin{aligned}
\left\langle f, S_{\alpha}^{* *} E\right\rangle & =\lim _{\beta}\left\langle S_{\alpha}^{*} f, \widehat{e}_{\beta}\right\rangle=\lim _{\beta}\left\langle m\left(e_{\alpha}, e_{\beta}-e_{\alpha} e_{\beta}\right), f\right\rangle \\
& =\lim _{\beta}\left\langle e_{\alpha}, m^{*}\left(f, e_{\beta}-e_{\alpha} e_{\beta}\right)\right\rangle=\left\langle m^{*}\left(f, e_{\alpha}\right), E-e_{\alpha} \cdot E\right\rangle \\
& =\left\langle e_{\alpha}, m^{* *}\left(E-e_{\alpha} \cdot E, f\right)\right\rangle=\left\langle f, m^{* * *}\left(\hat{e}_{\alpha}, E-e_{\alpha} \cdot E\right)\right\rangle
\end{aligned}
$$

which implies that $S_{\alpha *}^{* *} E=r^{* * *}\left(\widehat{e}_{\alpha x}, E-\widehat{e}_{\alpha}\right)$.

Now $e_{\alpha x} \cdot F \cdot \epsilon_{\alpha x} \cdot F \cdot e_{c}=e_{\alpha} \cdot m^{* * *}(E, E)-e_{\alpha} \cdot m^{* * *}(E, E) \cdot e_{\alpha}$ and since $m^{* * * *}$ is an olement of $Q M\left(A^{* * 4}, \cdot\right)$, we have

Thus

$$
e_{\alpha} \cdot F-e_{\alpha} \cdot F \cdot e_{\alpha \alpha}=m^{* * *}\left(\widehat{e}_{\alpha}, E-\widehat{e}_{\alpha}\right) \text {. }
$$

$$
S_{\alpha}^{* *} E=e_{\alpha} \cdot F-e_{\alpha} \cdot F \cdot e_{\alpha}
$$

From (3.3) and (3.4),

proving (ii).

$$
\left(\left(\lambda\left(S_{\alpha}\right)\right)^{\prime}\right)^{* * *}(E, E)=e_{\alpha} \cdot F-e_{\alpha} \cdot F \cdot e_{\alpha}
$$


Since $\left.a \cdot m^{* * *}(E, E) \cdot b=m^{* * *}(\widehat{a}, \widehat{b})=m \widehat{(a, b}\right)$, we have $F \in A_{\sigma}^{* *}$. Thus, by the proof of Lemma $3.4, e_{\alpha} \cdot F-e_{\alpha} \cdot F \cdot e_{\alpha}+F \cdot e_{\alpha}$ converges strictly to $F$; that is, for each $a \in A$,

(3.5) $\quad\left\|\left(\left(\left(\lambda\left(S_{\alpha}\right)\right)^{\prime}\right)^{* * *}(E, E)+\left(\varrho\left(T_{\alpha}\right)\right)^{* * *}(E, E)-m^{* * *}(E, E)\right) \cdot a\right\| \rightarrow 0$ and

(3.6) $\left\|a \cdot\left(\left(\left(\lambda\left(S_{\alpha}\right)\right)^{\prime}\right)^{* * *}(E, E)+\left(\varrho\left(T_{\alpha}\right)\right)^{* * *}(E, E)-m^{* * *}(E, E)\right)\right\| \rightarrow 0$.

Next we require the following identities:
(iii) $\left(m^{\prime}\right)^{* * *}(E, E) \cdot a=m^{* * *}(E, E) \cdot a$,
(iv) $(m \circ a)^{* * *}=m^{* * *} \circ \widehat{a}$
(v) $(a \circ m)^{* * *}=\widehat{a} \circ m^{* * *}$

( $m \in Q M(A), a \in A$ ). We prove (iii) below; (iv) and (v) can be proved using routine calculations and the property that $m^{* * *}$ is a quasi-multiplier on $\left(A^{* *}, \cdot\right)$. Since $\left(m^{\prime}\right)^{* * *}$ is a quasi-multiplier on $\left(A^{* *}, *\right)$,

$$
\begin{aligned}
\left\langle f,\left(m^{\prime}\right)^{* * *}(E, E) \cdot a\right\rangle & =\left\langle f,\left(m^{\prime}\right)^{* * *}(E, \widehat{a})\right\rangle \\
& =\left\langle a,\left(m^{\prime}\right)^{* *}(f, E)\right\rangle=\left\langle\left(m^{\prime}\right)^{*}(a, f), E\right\rangle \\
& =\left\langle m^{* *}(\widehat{a}, f), E\right\rangle=\left\langle f, m^{* * *}(E, \widehat{a})\right\rangle,
\end{aligned}
$$

which implies that

$$
\left(m^{\prime}\right)^{* * *}(E, E) \cdot a=m^{* * *}(E, E) \cdot a .
$$

It follows from (iv) that

$$
(m \circ a)^{* * *}(E, E)=\left(m^{* * *} \circ \widehat{a}\right)(E, E)=m^{* * *}(E, E) \cdot a,
$$

and from $(v)$ that

$$
(a \circ m)^{* * *}(E, E)=a \cdot m^{* * *}(E, E) .
$$

Thus, from (3.5), (iii), and the above we have

$$
\lim _{\alpha}\left\|\left(\left(\lambda\left(S_{\alpha}\right)+\varrho\left(T_{\alpha}\right)-m\right) \circ a\right)^{* * *}(E, E)\right\|=0 .
$$

It follows from Theorem 2.1 that

$$
\lim _{\alpha}\left\|\left(\lambda\left(S_{\alpha}\right)+\varrho\left(T_{\alpha}\right)-m\right) \circ a\right\|=0 .
$$

Similarly we can show that $\lim _{\alpha}\left\|a \circ\left(\lambda\left(S_{\alpha}\right)+\varrho\left(T_{\alpha}\right)-m\right)\right\|=0$. Thus $\lambda\left(S_{\alpha}\right)$ w$\varrho\left(T_{\alpha}\right)$ converges strictly to $m$; that is, $M_{l}(A)+M_{r}(A)$ is strictly deuse in $Q M(A)$.

4. Quasi-multipliers and the algebra of compact operators. Let $X$ be a Banach space and let $A=K_{0}(X)$, the algebra of bounded linear operators on $X$ which can be approximated, in the operator norm, by operators of finite rank. In this section our first aim is to establish a characterization for the quasi-multipliers of $A$. We begin, however, with some definitions and necessary background results. Full details of the results given may be found in [4]. A Banach space $X$ is said to have the approximation property if, for every compact set $K$ and $\varepsilon>0$, there exists a linear operator $T_{K, \varepsilon}$ of finite rank such that $\left\|T_{K, \varepsilon}(x)-x\right\|<\varepsilon$ for all $x \in K$. If, in addition, there exists a positive number $C$, inclependent of $K$ and $\varepsilon$, such that $\left\|T_{K, \varepsilon}\right\|<C$, then $X$ is said to have the bounded approximation property. If $C=1$, then $X$ is said to have the metric approximation property. If $X^{*}$ has the bounded approximation property, then $K_{0}(X)=K(X)$, the algebra of all compact operators on $X([4]$, Theorem 3.5$)$, and $K(X)$ has a bounded a.i.; the latter property follows from ([4], Theorems 3.10 and 3.11$),([4]$, p. 93$)$, and $([3]$, p. 59, Proposition 6).

The tensor $x \otimes x^{\prime}\left(x \in X, x^{\prime} \in X^{*}\right)$ determines a bounded linear operator on $X$ according to the equation

$$
\left(x \otimes x^{\prime}\right) y=\left(x^{\prime}(y)\right) x \quad(y \in X)
$$

and so the elements of the tensor product $X \otimes X^{*}$ are operators on $X$ which are of finite rank. If $u \in X \otimes X^{*}$ and $u=\sum_{i=1}^{n} x_{i} \otimes x_{i}^{\prime}$, the right hand side of the equation

$$
\|u\|^{\vee}=\sup \left\{\left|\sum_{i=1}^{n}\left\langle x_{i}, f\right\rangle\left\langle x_{i}^{\prime}, F\right\rangle\right|: f \in X^{*}, F \in X^{* *}\right\}
$$

is independent of the representation of $u$ and defines a norm on $X \otimes X^{*}$, called the inductive tensor norm. The completion of $X \otimes X^{*}$ with respect to $\|\cdot\|^{\vee}$ is denoted by $X \ddot{\otimes} X^{*}$ and it is straightforward to show that $X \ddot{\otimes} X^{*}$ is isometrically isomorphic to $K_{0}(X)$.

The projective tensor norm on $X \otimes X^{*}$ is defined by

$$
\|u\|^{\wedge}=\inf \left\{\sum_{i=1}^{n}\left\|x_{i}\right\|\left\|x_{i}^{\prime}\right\|: u=\sum_{i=1}^{n} x_{i} \otimes x_{i}^{\prime}\right\},
$$

where the infimum is taken over all representations of $u$. The completion of $X \otimes X^{*}$ with respect to $\|\cdot\|^{\wedge}$ is denoted by $X \hat{\otimes} X^{*}$ and is called the projective tensor product of $X$ and $X^{*}$. The mapping $\phi$ of $X \otimes X^{*}$ into $\mathcal{L}(X)$ as defined by $(4.1)$ is a norm decreasing mapping on $\left(X \otimes X^{*},\|\cdot\|^{\wedge}\right)$ and so induces a contraction from $X \hat{\mathcal{O}} X^{*}$ into $\mathcal{L}(X)$. Clearly its image is linearly isomorphic to the quotient space $X \hat{\otimes} X^{*} / \operatorname{ker} \phi ; \phi\left(X \hat{\otimes} X^{*}\right)$, equipped with the quotient norm, is denoted by $N(X)$. The elements of $N(X)$ are called the nuclear operators on $X$ and the norm is called the trace norm. In fact, if $u \in N(X)$, then the trace norm of $u$ is given by

$$
\|u\|_{N}=\inf \left\{\sum_{i=1}^{\infty}\left\|x_{i}\right\|\left\|x_{i}^{\prime}\right\|: u=\sum_{i=1}^{\infty} x_{i} \otimes x_{i}^{\prime}\right\},
$$

where the infimum is taken over all representations of $u$. 
A bounded linear operator $T$ on $X$ is said to be an integral operator if there exists a constant $C>0$ such that

$$
\left|\sum_{i=1}^{n}\left\langle T x_{i}, x_{i}^{\prime}\right\rangle\right| \leq C\left\|\sum_{i=1}^{n} x_{i} \otimes x_{i}^{\prime}\right\|
$$

for all $\sum_{i=1}^{n} x_{i} \otimes x_{i}^{\prime} \in X \otimes X^{*}$, where $\|\cdot\|$ denotes the operator norm on $\mathcal{L}(X)$. The infimum over all possible constants $C$ is called the integral norm of $T$ and is denoted by $\|T\|_{I}$. If $I(X)$ denotes the integral operators on $X$, then $\left(I(X),\|\cdot\|_{I}\right)$ is a Banach space $([23]$, p. 258). Every nuclear operator is integral, its integral norm being dominated by its nuclear norm. The dual of $K_{0}(X)$ is isometrically isomorphic to $\left(I\left(X^{*}\right),\|\cdot\|_{I}\right)$; the correspondence $F \leftrightarrow f$ between $\left(K_{0}(X)\right)^{*}$ and $I\left(X^{*}\right)$ is described by the relation

$$
\langle u, F\rangle=\sum_{i=1}^{n}\left\langle x_{i}, f x_{i}^{\prime}\right\rangle \quad\left(f \in I\left(X^{*}\right)\right)
$$

where $u=\sum_{i=1}^{n} x_{i} \otimes x_{i}^{\prime} \in X \otimes X^{*}$.

For our investigations in this section we require, in addition to the above, the following properties of integral operators.

(i) $I(X)$ is a two-sided ideal of $\mathcal{L}(X)$ and, for $f, g \in I(X), T \in \mathcal{L}(X)$ $\|f \circ T \circ g\|_{I} \leq\|f\|_{I}\|T\|\|g\|_{I}$; the proof is routine.

(ii) An operator $f$ on $X$ is integral if and only if $f^{*}$ is integral and $\|f\|_{I}=\left\|f^{*}\right\|_{I}([5]$, p. 236, Corollary 11$)$.

(iii) An integral operator on $X$ is weakly compact ([4], p. 228, Corollary 3.6) and so $f^{* *}$ maps $X^{* *}$ into $\widehat{X}([4]$, p. 227).

The bilinear functional $\left(x^{\prime}, x^{\prime \prime}\right) \rightarrow\left\langle x^{\prime}, x^{\prime \prime}\right\rangle$ on $X^{*} \times X^{* *}$ induces a unique linear functional $\psi$ on $X^{*} \otimes X^{* *}$ such that

$$
\psi\left(\sum_{i=1}^{n} x_{i}^{\prime} \otimes x_{i}^{\prime \prime}\right)=\sum_{i=1}^{n}\left\langle x_{i}^{\prime}, x_{i}^{\prime \prime}\right\rangle
$$

([3], p. 232, Theorem 6). It is clear that $\psi$ is a continuous linear functional on $\left(X^{*} \otimes X^{* *},\|\cdot\|^{\wedge}\right)$ and so has a unique continuous extension, $\tilde{\psi}$ say, to $X^{*} \hat{\otimes} X^{* *}$. Since the right hand side of $(4.3)$ is independent of the representation of $u=\sum_{i=1}^{n} x_{i}^{\prime} \otimes x_{i}^{\prime \prime}$, we refer to it as the trace of $u$ and it is written tr.u. In particular, if $g \in \mathcal{L}\left(X^{*}\right)$, then $g \circ u=\sum_{i=1}^{n} g x_{i}^{\prime} \otimes x_{i}^{\prime \prime}$, and so

$$
\operatorname{tr} .(g \circ u)=\sum_{i=1}^{n}\left\langle g x_{i}^{\prime}, x_{i}^{\prime \prime}\right\rangle=\sum_{i=1}^{n}\left\langle x_{i}^{\prime}, g^{*} x_{i}^{\prime \prime}\right\rangle=\operatorname{tr} .(u \circ g) .
$$

If $f \in I\left(X^{*}\right)$ and $a=x \otimes x^{\prime} \in X \otimes X^{*}$, then $a^{*}=x^{\prime} \otimes \widehat{x}$ and so tr. $\left(a^{*} \circ f\right)=$ $\left\langle x^{\prime}, f^{*} \widehat{x}\right\rangle=\left\langle x, f x^{\prime}\right\rangle=\operatorname{tr} .\left(f \circ a^{*}\right)=\langle a, f\rangle$ (by (4.2)). It follows that, for any $a \in K_{0}(X)$

$$
\operatorname{tr} .\left(f \circ a^{*}\right)=\operatorname{tr} .\left(a^{*} \circ f\right)=\langle a, f\rangle .
$$

We let $\pi$ denote the canonical mapping of $X^{*} \hat{\otimes} X^{* *}$ into $I\left(X^{*}\right)$ (in fact, the image of $X^{*} \hat{\otimes} X^{* *}$ under $\pi$ is $N\left(X^{*}\right)$ ). If $X^{*}$ has the bounded approximation property, then $\pi$ is injective ([4], p. 80, Theorem 3.4), so that $N\left(X^{*}\right)$ is the projective tensor product $X^{*} \hat{\otimes} X^{* *}$ of $X^{*}$ and $X^{* *}$. In this case tr. $u$ is well defined for every $u \in N\left(X^{*}\right)$. The dual space $\left(X^{*} \dot{\otimes} X^{* *}\right)^{*}$ may be identified (isometrically and isomorphically) with $\mathcal{L}\left(X^{* *}\right)$, the correspondence $h \rightarrow T_{h}\left(h \in\left(X^{*} \hat{\otimes} X^{* *}\right)^{*}, T_{h} \in \mathcal{L}\left(X^{* *}\right)\right)$ being defined by the relation

$$
\left\langle x^{\prime} \otimes x^{\prime \prime}, h\right\rangle=\left\langle x^{\prime}, T_{h} x^{\prime \prime}\right\rangle \text {. }
$$

Thus the adjoint $\pi^{*}$ is a mapping of $\left(K_{0}(X)\right)^{* *}$ into $\mathcal{L}\left(X^{* *}\right)$.

Lemma 4.1. Let $X$ be a Banach space and suppose that $a \in K_{0}(X)$. Then $\pi^{*} \widehat{a}=a^{* *}$.

Proof. Let $x^{\prime} \in X^{*}, x^{\prime \prime} \in X^{* *}$. It follows from (4.4) that, for any $a \in K_{0}(X)$,

$$
\begin{aligned}
\left\langle x^{\prime},\left(\pi^{*} \widehat{a}\right) x^{\prime \prime}\right\rangle & =\left\langle x^{\prime} \otimes x^{\prime \prime}, \pi^{*} \widehat{a}\right\rangle=\left\langle\pi\left(x^{\prime} \otimes x^{\prime \prime}\right), \widehat{a}\right\rangle \\
& =\left\langle a, \pi\left(x^{\prime} \otimes x^{\prime \prime}\right)\right\rangle=\operatorname{tr} .\left(a^{*} \circ \pi\left(x^{\prime} \otimes x^{\prime \prime}\right)\right) \\
& =\operatorname{tr} .\left(\pi\left(a^{*} x^{\prime} \otimes x^{\prime \prime}\right)\right)=\left\langle a^{*} x^{\prime}, x^{\prime \prime}\right\rangle=\left\langle x^{\prime}, a^{* *} x^{\prime \prime}\right\rangle,
\end{aligned}
$$

which implies that $\left(\pi^{*} \widehat{a}\right)=a^{* *}$.

For the remainder of this section, unless stated otherwise, we assume that $X^{*}$ has the bounded approximation property and in the sequel the algebra $K_{0}(X)$ is denoted by $A$. We also use $\iota_{X}$ (resp. $\iota_{X^{*}}$ ) to denote the canonical embedding of $X$ (resp. $X^{*}$ ) into $X^{* *}$ (resp. $X^{* * *}$ ) and, for each $h \in \mathcal{L}\left(X^{* *}\right)$, the "h-flat" mapping is the element of $\mathcal{L}\left(X^{*}\right)$ defined by $h^{b}=\iota_{X}^{*} \circ h^{*} \circ \iota_{X}$.

TheOrem 4.2. Let $X$ be a Banach space and suppose that $X^{*}$ has the bounded approximation property. Then $\varrho$ is a topological isomorphism of $M_{r}(A)$ onto $Q M(A)$.

Proof Since $X^{m}$ has the bounded approximation property, $A$ has a bounded two-sided approximate identity and so $\varrho$ is a topological isomorphism (isometric if $X^{*}$ has the metric approximation property). Thus to complete the proof we have to show that $\varrho$ is onto.

Let $m \in Q M(A)$ and let $E$ be a right (left) identity with respect to the first (second) Arens product on $A^{* *}$. Let $F=m^{* * *}(E, E)$ and $g=\left(\pi^{*} F\right)^{\text {b: }}$ The algebra $M_{r}(A)$ is isometrically isomorphic to $\mathcal{L}\left(X^{*}\right)$ (see, for example, $([10]$, Theorem 19 and Corollary 1$)$ ), and the correspondence $\tau$ which maps $g \in \mathcal{L}\left(X^{*}\right)$ onto $\tau_{g} \in M_{r}(A)$ has the property that $\iota_{X} \circ \tau_{g}(a)=a^{* *} \circ g^{*} \circ \iota \mathrm{X}$ 
for all $a \in A$. Thus, for any $a, b \in A, x \in X, x^{\prime} \in X^{*}$, we have

$$
\begin{aligned}
\left\langle x^{\prime}, \iota_{X}\left(\left(\tau_{g}(a) b\right) x\right)\right\rangle & =\left\langle x^{\prime},\left(a^{* *} \circ g^{*}\right) \widehat{b x}\right\rangle \\
& =\left\langle x^{\prime}, a^{* *} \circ\left(\pi^{*} F\right)^{b *}(\widehat{b x})\right\rangle,
\end{aligned}
$$

and since $\left.\left(h^{b}\right)^{*}\right|_{\widehat{X}}=h$ for every $h \in \mathcal{L}\left(X^{* *}\right)$, it follows that

$$
\begin{aligned}
\left\langle x^{\prime}, \iota_{X}\left(\left(\tau_{g}(a) b\right) x\right)\right\rangle & =\left\langle x^{\prime},\left(a^{* *} \circ \pi^{*} F \circ b^{* * *} \circ \iota_{X}\right) x\right\rangle \\
& =\left\langle x^{\prime},\left(\pi^{*} \widehat{a} \circ \pi^{*} F \circ \pi^{*} \hat{b} \circ \iota_{X}\right) x\right\rangle
\end{aligned}
$$

by Lemma 4.1 .

The mapping $\pi^{*}$ satisfies $\pi^{*}(F \cdot G)=\pi^{*} F \circ \pi^{*} G$ for all $F, G \in A^{* *}$ ([9], Proposition 3.2(iii)) and so

$$
\begin{aligned}
\left\langle x^{\prime}, \iota_{X}\left(\left(\tau_{g}(a) b\right) x\right)\right\rangle & =\left\langle x^{\prime}, \pi^{*}\left(a \cdot m^{* * *}(E, E) \cdot b\right) \widehat{x}\right\rangle \\
& =\left\langle x^{\prime},\left(\pi^{*}\left(m^{* * *}(\widehat{a}, \widehat{b})\right)\right) \widehat{x}\right\rangle=\left\langle x^{\prime},\left(\pi^{*}(m \widehat{(a, b)})\right) \widehat{x}\right\rangle \\
& =\left\langle x^{\prime},(m(a, b))^{* *} \widehat{x}\right\rangle=\left\langle x^{\prime},(m \widehat{(a, b)}) x\right\rangle,
\end{aligned}
$$

which implies that $\tau_{g}(a) b=m(a, b)$; that is, $\varrho\left(\tau_{g}\right)=m$, proving that $\varrho$ is surjective, as required.

Corollary 4.3. Let $X$ be a Banach space and suppose that $X^{*}$ has the bounded approximation property. Then $Q M(A)$ is topologically isomorphic to $\mathcal{L}\left(X^{*}\right)$; the topological isomorphism is an isometric one if $X^{*}$ has the metric approximation property.

Corollary 4.4 (Vasudevan and Goel ([22], Lemma 3.2)). If $H$ is a Hilbert space and $A=K(H)$, then $\mathcal{L}(H)$ and $Q M(A)$ are isometrically isomorphic.

Pro of. The result follows immediately from Corollary 4.3 since every Hilbert space has the metric approximation property.

Before our next result we explain how we consider left, right and double multipliers of $A^{* *}$; our approach is due to Grosser ([9], p. 54.7). Suppose that $\mathcal{V}$ is a left module over a Banach algebra $\mathcal{A}$; that is, $\mathcal{V}$ is a Banach space and there is a continuous bilinear mapping $\mathcal{A} \times \mathcal{V} \rightarrow \mathcal{V}$ such that $a(b v)=$ $(a b) v(a, b \in \mathcal{A}, v \in \mathcal{V})$. A right multiplier of $\mathcal{V}$ is defined to be a mapping $T: \mathcal{A} \rightarrow \mathcal{V}$ such that $T(a b)=a T b$ for all $a, b \in \mathcal{A}$. If $\mathcal{A}$. has a bounded right a.i., then every right multiplier is linear and continuous. Following the notation introduced in $\S 2$ we denote the set of all linear and continuous right multipliers of $\mathcal{V}$ by $M_{r}(\mathcal{V})$. The space $M_{l}(\mathcal{W})$ of all linear and continuous left multipliers of a right $\mathcal{A}$-module $\mathcal{W}$ is defined analogously. If $\mathcal{V}$ is an $\mathcal{A}$-bi-module (that is, a left and a right $\mathcal{A}$-module simultaneously satisfying $a(v b)=(a v) b$ for all $a, b \in \mathcal{A}$ and $v \in \mathcal{V})$, then the space $M(\mathcal{V})$ of continuous linear double multipliers of $\mathcal{V}$ consists of all pairs $(S, T), S \in M_{l}(\mathcal{V})$ and
$T \in M_{r}(\mathcal{V})$, satisfying $a S b=(T a) b(a, b \in \mathcal{A})$. In particular, $\mathcal{A}^{* *}$ is an $\mathcal{A}$-bi-module and so the right, left and double multipliers of $\mathcal{A}^{* *}$ are defined in the above sense. If $S$ is a linear continuous mapping of $\mathcal{A}$ into $\mathcal{A}^{* *}$ and $S^{t}$ denotes the restriction of its adjoint to $\mathcal{A}^{*}$, then it is not difficult to show that $S$ is a left multiplier of $\mathcal{A}^{* *}$ if and only if $S^{t}(a * f)=a * S^{t} f$ for all $a \in \mathcal{A}, f \in \mathcal{A}^{*}$. Similarly, a continuous linear operator $T: \mathcal{A} \rightarrow \mathcal{A}^{* *}$ is a right multiplier of $\mathcal{A}^{* *}$ if and only if $T^{t}(f \cdot a)=T^{t} f \cdot a$ for all $a \in \mathcal{A}$ and $f \in \mathcal{A}^{*}$, and the pair $(S, T)$ (where $S, T: \mathcal{A} \rightarrow \mathcal{A}^{* *}$ are continuous linear mappings) is a clouble multiplier of $\mathcal{A}^{* *}$ if and only if $\left\langle b, S^{t}(f \cdot a)\right\rangle=\left\langle a, T^{t}(b * f)\right\rangle$ for all $a, b \in A$, and $f \in \mathcal{A}^{*}$. Thus we may regard $M_{l}\left(\mathcal{A}^{* *}\right)$ and $M_{r}\left(\mathcal{A}^{* *}\right)$ as subspaces of $\mathcal{L}\left(\mathcal{A}^{*}\right)$ and $M\left(\mathcal{A}^{* *}\right)$ as a subspace of $\mathcal{L}\left(\mathcal{A}^{*}\right) \times \mathcal{L}\left(\mathcal{A}^{*}\right)$.

We now return to the algebra $A$ and to the spaces $M_{r}\left(A^{* *}\right)$ and $M_{l}\left(A^{* *}\right)$. In ([8], Theorem 1) Grosser proved that $M_{r}\left(A^{* *}\right)$ is isometrically isomorphic to $\mathcal{L}\left(X^{* * *}\right)$ and that $M_{l}\left(A^{* *}\right)$ is isometrically isomorphic to $\mathcal{L}\left(X^{*}\right)$. We note that the characterizations do not require $X^{*}$ to have the bounded approximation property and are therefore valid for any Banach space $X$ and $A=K_{0}(X)$. For the sake of completeness we give the results in the following theorem using the notation and terminology developed in this paper.

THEOREM (Grosser [8], Theorem 1). Let $X$ be a Banach space and $A=$ $K_{0}(X)$

(a) The mapping $\sigma^{\prime}: \mathcal{L}\left(X^{*}\right) \rightarrow M_{l}\left(A^{* *}\right)$, which acts on $A^{*}$ according to the equation

$$
\sigma_{g}^{\prime}(f)=g \circ f \quad\left(g \in \mathcal{L}\left(X^{*}\right), f \in A^{*}\right)
$$

is an isometric isomorphism of $\mathcal{L}\left(X^{*}\right)$ onto $M_{l}\left(A^{* *}\right)$.

(b) For each $h \in \mathcal{L}\left(X^{* *}\right)$ and $f \in A^{*},\left(h \circ f^{*}\right)^{b} \in A^{*}$. Consequently, the mapping $\tau^{\prime}: \mathcal{L}\left(X^{* *}\right) \rightarrow M_{r}\left(A^{* *}\right)$, whose action on $A^{*}$ is given by the equation

$$
\tau_{h}^{\prime}(f)=\left(h \circ f^{*}\right)^{b},
$$

is an isometric isomorphism of $\mathcal{L}\left(X^{* *}\right)$ onto $M_{r}\left(A^{* *}\right)$.

The isometric isomorphism between $A^{*}$ and the Banach algebra $\left(I\left(X^{*}\right),\|\cdot\| r\right)$ of integral operators on $X^{*}$ enables us to define a product on $A^{*}$ which makes it a Banach algebra. Consequently; we can consider mappings $q: A^{*} \times A^{*} \rightarrow A^{*}$ which satisfy the quasi-multiplier condition (2.1). As in $\S 2$, we let $\overrightarrow{Q M}\left(A^{*}\right)$ clenote the space of all jointly continuous quasi-multipliers on $A^{*}$.

THEOREM 4.5. Let $X$ be a Banach space and $A=K_{0}(X)$, Then the equation

$$
(\theta(h))(f, g)=\left(\tau_{h}^{\prime} f\right) \odot g \quad\left(h \in \mathcal{L}\left(X^{*}\right), f, g^{*} \in A^{*}\right)
$$


defines a norm decreasing linear isomorphism between $\mathcal{L}\left(X^{* *}\right)$ and a subspace of $Q M\left(A^{*}\right)$.

Proof. We recall that $A^{*} \simeq\left(I\left(X^{*}\right),\|\cdot\|_{I}\right)$, the integral operators on $X^{*}$, and $I\left(X^{*}\right)$ is a Banach algebra with respect to the integral norm. We first show that $\theta$ maps $\mathcal{L}\left(X^{* *}\right)$ into $Q M\left(A^{*}\right)$. Let $h \in \mathcal{L}\left(X^{* *}\right)$ and $f \in A^{*}$. We first note that $\left(\tau_{h}^{\prime} f\right)^{*}=h \circ f^{*}$; for if $x^{\prime} \in X^{*}, x^{\prime \prime} \in X^{* *}$, then

$$
\begin{aligned}
\left\langle x^{\prime},\left(\tau_{h}^{\prime} f\right)^{*} x^{\prime \prime}\right\rangle & =\left\langle\left(\tau_{h}^{\prime} f\right) x^{\prime}, x^{\prime \prime}\right\rangle \\
& =\left\langle x^{\prime \prime},\left(\iota_{X^{*}} \circ \iota_{X}^{*} \circ f^{* *} \circ h^{*} \circ \iota_{X}\right) x^{\prime}\right\rangle \\
& =\left\langle x^{\prime \prime},\left(h \circ f^{*}\right)^{*} \widehat{x^{\prime}}\right\rangle \quad\left(\text { since } \iota_{X^{*}} \circ \iota_{X}^{*}=\mathrm{id} . \widehat{X}^{*}\right) \\
& =\left\langle x^{\prime},\left(h \circ f^{*}\right) x^{\prime \prime}\right\rangle,
\end{aligned}
$$

which implies that $\left(\tau_{h}^{\prime} f\right)^{*}=h \circ f^{*}$. Thus, for $f, g \in A^{*}$,

$$
\left(\tau_{h}^{\prime}(f \circ g)\right)^{*}=h \circ g^{*} \circ f^{*}=\left(f \circ \tau_{h}^{\prime}(g)\right)^{*},
$$

and so $\tau_{h}^{\prime}(f \circ g)=f \circ \tau_{h}^{\prime} g$. It follows that, for $f, g, l \in A^{*}$,

$$
\theta(h)(f \circ g, l)=\tau_{h}^{\prime}(f \circ g) \circ l=f \circ \tau_{h}^{\prime} g \circ l=f \circ \theta(h)(g, l) .
$$

Similarly, we can show that $\theta(h)(f, g \circ l)=\theta(h)(f, g) \circ l$, so that $\theta(h) \in$ $Q M\left(A^{*}\right)$. The linearity of $\theta$ follows immediately from the linearity of $\tau^{\prime}$. We show that $\theta$ is continuous, as follows:

$$
\begin{aligned}
\|\theta(h)(f, g)\|_{I} & =\left\|(\theta(h)(f, g))^{*}\right\|_{I}=\left\|\left(\tau_{h}^{\prime} f \circ g\right)^{*}\right\|_{I} \\
& =\left\|g^{*} \circ h \circ f^{*}\right\|_{I} \leq\left\|g^{*}\right\| I\|h\|\left\|f^{*}\right\|_{I}=\|g\|_{I}\|f\|_{I}\|h\|,
\end{aligned}
$$

which implies that $\theta$ is continuous, with $\|\theta\| \leq 1$.

Finally, $\theta$ is injective. Suppose that $\theta(h)=0$. Then $\tau_{h}^{\prime} f \circ g=0$ for all $f, g \in A^{*}$. In particular, for any $x^{\prime} \in X$ and $x^{\prime \prime} \in X^{* *}, \tau_{h}^{\prime} f \circ \pi\left(x^{\prime} \otimes x^{\prime \prime}\right)=0$. Thus, for any $y^{\prime} \in X^{*}$,

$$
0=\left(\tau_{h}^{\prime} f \circ \pi\left(x^{\prime} \otimes x^{\prime \prime}\right)\right) y^{\prime}=\left\langle y^{\prime}, x^{\prime \prime}\right\rangle\left(\tau_{h}^{\prime} f\right) x^{\prime},
$$

which implies that, since $x^{\prime}, y^{\prime}$ and $x^{\prime \prime}$ are arbitrary, $\tau_{h}^{\prime}=0$. Since $\tau^{\prime}$ is an isomorphism, $h=0$, as required.

DEFINITION 4.6. Let $\mathcal{A}$ be a Banach algebra with a bounded approximate identity. A mapping $m: \mathcal{A} \times \mathcal{A} \rightarrow \mathcal{A}^{* *}$ is said to be a quasi-multiplier of $\mathcal{A}^{* *}$ if

$$
m(a b, c)=\widehat{a} \cdot m(b, c) \quad \text { and } \quad m(a, b c)=m(a, b) \cdot \widehat{c} \quad \text { for all } a, b, c \in \mathcal{A} \text {. }
$$

Let $Q M\left(\mathcal{A}^{* *}\right)$ denote the set of all bilinear and jointly continuous quasimultipliers of $\mathcal{A}^{* *}$. Then, as in the case of quasi-multipliers on $\mathcal{A}$, every quasi-multiplier of $\mathcal{A}^{* *}$ belongs to $Q M\left(\mathcal{A}^{* *}\right)$ and $Q M\left(\mathcal{A}^{* *}\right)$ is a Banach space. We note that $Q M(\mathcal{A})$ is the subspace of $Q M\left(\mathcal{A}^{* *}\right)$ which consists of those $m \in Q M\left(\mathcal{A}^{* *}\right)$ such that $m(\mathcal{A}, \mathcal{A}) \subseteq \widehat{\mathcal{A}}$.
We extend $m \in Q M\left(\mathcal{A}^{* *}\right)$ to a linear map on $\mathcal{A}^{* *} \times \mathcal{A}^{* *}$ in the following way:

$m^{*}: \mathcal{A}^{* * *} \times \mathcal{A} \rightarrow \mathcal{A}^{*}$, defined by $\left\langle b, m^{*}(\mathcal{F}, a)\right\rangle=\langle m(a, b), \mathcal{F}\rangle$,

$m^{* *}: \mathcal{A}^{* *} \times \mathcal{A}^{* * *} \rightarrow \mathcal{A}^{*}$, defined by $\left\langle a, m^{* *}(F, \mathcal{F})\right\rangle=\left\langle m^{*}(\mathcal{F}, a), F\right\rangle$ $m^{* * *}: \mathcal{A}^{* *} \times \mathcal{A}^{* *} \rightarrow \mathcal{A}^{* * * *}$, defined by $\left\langle\mathcal{F}, m^{* * *}(F, G)\right\rangle=\left\langle m^{* *}(G, \mathcal{F}), F\right\rangle$

$\left(a, b \in \mathcal{A}, F, G \in \mathcal{A}^{* *}, \mathcal{F} \in \mathcal{A}^{* * *}\right)$.

We use the notation to denote the first Arens product on the algebra $\mathcal{A}^{* *}$ and for convenience we also use $\cdot$ to denote the corresponding first Arens product on the algebra $\mathcal{A}^{* * * *}$.

Lemma 4.7. For $a, b \in \mathcal{A}, \widehat{\widehat{a}} \cdot m^{* * *}(E, E) \cdot \widehat{\widehat{b}} \in \widehat{\mathcal{A}^{* *}}$.

Proof. Let $\mathcal{F}$ be any element of $\mathcal{A}^{* * *}$. Then

$$
\begin{aligned}
& \left\langle\mathcal{F}, \widehat{\widehat{a}} \cdot m^{* * *}(E, E) \cdot \widehat{\widehat{b}}\right\rangle=\left\langle\widehat{\hat{b}} \cdot \mathcal{F} \cdot \widehat{a}, m^{* * *}(E, E)\right\rangle \\
& =\left\langle m^{* *}(E, \widehat{\hat{b}} \cdot \mathcal{F} \cdot \widehat{a}), E\right\rangle=\lim _{\alpha}\left\langle e_{\alpha}, m^{* *}(E, \widehat{\hat{b}} \cdot \mathcal{F} \cdot \widehat{a})\right\rangle \\
& =\lim _{\alpha}\left\langle m^{*}\left(\widehat{\hat{b}} \cdot \mathcal{F} \cdot \widehat{a}, e_{\alpha}\right), E\right\rangle=\lim _{\alpha} \lim _{\beta}\left\langle e_{\beta}, m^{*}\left(\widehat{\widehat{b}} \cdot \mathcal{F} \cdot \widehat{a}, e_{\alpha}\right)\right\rangle \\
& =\lim _{\kappa} \lim _{\beta}\left\langle m\left(e_{\alpha}, e_{\beta}\right), \widehat{\hat{b}} \cdot \mathcal{F} ; \widehat{a}\right\rangle=\lim _{\alpha} \lim _{\beta}\left\langle\widehat{\widehat{b}} \cdot \mathcal{F}, \widehat{\widehat{a}} \cdot m\left(\widehat{e_{\alpha}, e_{\beta}}\right)\right\rangle \\
& =\lim _{\alpha} \lim _{\beta}\left\langle\mathcal{F}, \widehat{\hat{a}} \cdot m\left(\widehat{e_{\alpha}, e_{\beta}}\right) \cdot \widehat{\hat{b}}\right\rangle=\lim _{\alpha} \lim _{\beta}\left\langle\mathcal{F}, \iota_{\mathcal{A}^{* *}}\left(\widehat{a} \cdot m\left(e_{\alpha}, e_{\beta}\right) \cdot \widehat{b}\right)\right\rangle \\
& =\lim _{c} \lim _{\beta}\left\langle\mathcal{F}, \iota_{\mathcal{A}^{* *}}\left(m\left(a e_{\alpha}, e_{\beta} b\right)\right)\right\rangle=\langle\mathcal{F}, \widehat{m(a, b)}\rangle
\end{aligned}
$$

which implies that $\widehat{\hat{a}} \cdot m^{* * *}(E, E) \cdot \hat{\hat{b}}=\widehat{m(a, b)} \in \widehat{\mathcal{A}^{* *}}$, as required.

THEOREM 4.8. Let $X$ be a Banach space and suppose that $X^{*}$ has the bounded approximation property. Then the mapping $\lambda: M_{l}\left(A^{* *}\right) \rightarrow$ $Q M\left(A^{* * *}\right)$, defined by

$$
\lambda(S)(a, b)=\widehat{a} \cdot S b \quad\left(S \in M_{l}\left(A^{* *}\right), a, b \in A\right),
$$

is a norm decreasing linear mapping of $M_{l}\left(A^{* *}\right)$ onto $Q M\left(A^{* *}\right)$.

Proof. It is clear that $\lambda$ is linear and norm decreasing. Therefore to complete the proof it is enough to show that $\lambda$ is a surjection.

Let $F=\left.\left(m^{* * *}(E, E)\right)\right|_{A^{*}}$. In the sequel we regard $F$ as an element of $A^{* *}$. Let $g=\left(\pi^{*} F\right)^{b}$. Then $g \in \mathcal{L}\left(X^{*}\right)$; we recall that $\mathcal{L}\left(X^{*}\right)$ is isometrically isomorphic to $M_{l}\left(A^{* *}\right)$, the isomorphism $\sigma^{\prime}$ being given by $\sigma_{g}^{\prime}(f)=g \circ f$ $\left(g \in \mathcal{L}\left(X^{*}\right), f \in A^{*}\right)$. It follows that the identity

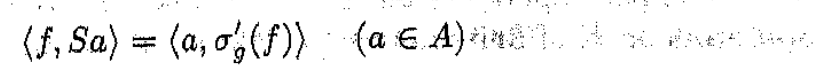


defines an element $S$ of $M_{l}\left(A^{* *}\right)$; for,

$$
\begin{aligned}
\langle f, S(a b)\rangle & =\left\langle a b, \sigma_{g}^{\prime}(f)\right\rangle=\left\langle a, b * \sigma_{g}^{\prime}(f)\right\rangle \\
& =\left\langle a, \sigma_{g}^{\prime}(b * f)\right\rangle=\langle b * f, S a\rangle=\langle b, f * S a\rangle,
\end{aligned}
$$

which implies that $S(a b)=S a * \widehat{b}$.

Thus, for all $a, b \in A, f \in A^{*}$,

$$
\begin{aligned}
\langle f, \widehat{a} \cdot S b\rangle & =\langle f \cdot a, S b\rangle=\left\langle b, \sigma_{g}^{\prime}(f \cdot a)\right\rangle \\
& =\left\langle b,\left(\pi^{*} F\right)^{b} \circ(f \cdot a)\right\rangle \\
& =\langle b,(f \cdot a) * F\rangle \quad([9], \text { Proposition 3.2(ii)) } \\
& =\langle b *(f \cdot a), F\rangle=\langle\widehat{b} \cdot(f \cdot a), F\rangle \\
& =\langle f, \widehat{a} \cdot F \cdot \widehat{b}\rangle=\left\langle\widehat{f}, \widehat{a} \cdot m^{* * *}(E, E) \cdot \widehat{\widehat{b}}\right\rangle \\
& =\langle\widehat{f}, \widehat{m(a, b)}\rangle \quad \text { by Lemma } 4.7 \\
& =\langle f ; m(a, b)\rangle,
\end{aligned}
$$

which implies that $a \cdot S b=m(a, b)$. It follows that $\lambda(S)=m$, as required.

Rem ark. If $E$ is an identity for the first Arens product (this is the case if, for example, $N\left(X^{*}\right)=I\left(X^{*}\right)$; see $([9]$, p. 560)), then $\lambda$ is a topological isomorphism. For, in this case, $\lambda(S)\left(e_{\alpha}, a\right)=\widehat{e}_{\alpha} \cdot S_{a} \vec{\alpha} E \cdot S_{a}=S_{a}$ for all $a \in$ $A$, which implies that $\lambda$ is injective and $\lambda^{-1}$ is continuous. If, in addition, $X^{*}$ has the metric approximation property, then $\lambda$ is an isometric isomorphism. Moreover, since $\mathcal{L}\left(X^{* *}\right) \cong M_{l}\left(A^{* *}\right)$ we have $\mathcal{L}\left(X^{* *}\right) \cong Q M\left(A^{* *}\right)$ in this case.

5. Quasi-multipliers of the Pedersen ideal. Let $A$ be a $C^{*}$-algebra and $\widetilde{A}$ denote the $C^{*}$-algebra obtained by adjoining the identity 1 to $A$. An element $a \in A$ is said to be positive if it is self-adjoint and $\operatorname{Sp}(a) \subseteq \mathbb{R}_{+}$ $(\operatorname{Sp}(a)=\{\lambda \in \mathbb{C}: a-\lambda 1$ is singular in $\widetilde{A}\})$; the set of positive elements of $A$ is denoted by $A^{+}$. A subcone $J$ of $A^{+}$is said to be an order ideal if the condition $x \leq y$ for $y \in J$ and $x \in A^{+}$implies that $x \in J$. An order ideal $J$ of $A^{+}$is said to be invariant if $a^{*} J a \subseteq J$ for all $a \in A$. A *-subalgebra $B$ of $A$ is said to be order-related if $B^{+}$is an order ideal in $A^{+}$and $B$ is the linear span of $B^{+}$. In ([16], Theorem 1.3), Pedersen proved that every $C^{*}$ walgebra contains a minimal, dense, order-related, two-sided ideal $K_{A}$. This ideal has subsequently become known as the Pedersen $i d e a l$. If $X$ is a locally compact Hausdorff space and $A=C_{0}(X)$, the complex-valued functions on $X$ which vanish at infinity, then $K_{A}=C_{00}(X)$, the functions in $C_{0}(X)$ which have compact supports $([7]$, p. $109,7 \mathrm{E}$ and $7 \mathrm{~F})$. If $A=\mathcal{B}_{0}(\mathcal{H})$, the $C^{*}$-algebra of all compact operators on a Hilbert space $\mathcal{H}$, then $K_{A}=\mathcal{B}_{00}(\mathcal{H})$, the operators on $\mathcal{H}$ of finite rank ([15], Theorem 2.4.7 and Theorem 3.3.3).
LemMa 5.1. Let $A$ be a $C^{*}$-algebra and let $K_{A}$ denote its Pedersen ideal. If $\left\{x_{i}\right\}$ is a finite set of elements in $K_{A}$, then the order-related $C^{*}$-algebra generated by them is contained in $K_{A}$.

Proof. See ([16], Proposition 4).

We shall require the following version of the Cohen-Hewitt factorization theorem.

Theorem 5.2. Let $A$ be a $C^{*}$-algebra and let $\left\{x_{i}: i=1, \ldots, n\right\}$ be a finite set of elements in $K_{A}$. Then, for each $\varepsilon>0$, there exist elements $y_{1}, \ldots, y_{n}, z_{1}, \ldots, z_{n}$ in $K_{A}$ and $a, b \in K_{A}^{+}$such that

$$
\left\|x_{i_{i}}-y_{i}\right\|<\varepsilon, \quad\left\|x_{i}-z_{i}\right\|<\varepsilon,
$$

and $x_{i}=a y_{i}=z_{i} b$ for $i=1, \ldots, n$.

Proof. Let $B$ be the order-related $C^{*}$-algebra generated by $\left\{x_{i}: i=\right.$ $1, \ldots, n\}$. By Lemma 5.1, $B \subseteq K_{A}$. Every $C^{*}$-algebra has a bounded a.i. consisting of positive elements (see, for example, the proof of ([18], p. 11, Theorem 1.4.2) and so, without loss of generality, we may assume that $B$ contains an a.i. $\left\{e_{\alpha x}: \alpha \in I\right\}$ consisting of elements in $B^{+}$. By the CohenHewitt factorization theorem there exist elements $y_{1}, \ldots, y_{n}, z_{1}, \ldots, z_{n}$, and $a, b$ in $B$ such that $x_{i}=a y_{i}=z_{i} b$ and

$$
\left\|x_{i}-y_{i}\right\|<\varepsilon, \quad\left\|x_{i}-z_{i}\right\|<\varepsilon \quad\left(i=1, \ldots, n_{i}\right) .
$$

A close examination of the proof of the Cohen-Hewitt factorization (as given, for example, in ([6], Theorem 16.1, p. 93 et seq.)) shows that $a$ (resp. $b)$ is the limit of a sequence of elements in $B^{+}$, and so, since $B^{+}$is closed in $B, a$ (resp. $b) \in B^{+}$. Since $B^{+} \subseteq K_{A}^{+}$, the proof is complete.

A quasi-multiplier on $K_{A}$ is a mapping of $K_{A} \times K_{A} \rightarrow K_{A}$ which satisfies conditions (2.1). The Pedersen ideal is not in general a Banach algebra and we cannot therefore make a direct appeal to ([14], Theorem 1) to deduce that the quasi-multipliers on $K_{A}$ are (i) bilinear and (ii) jointly continuous. However, an application of Theorem 5.2 enables us to establish (i) as follows.

Let $m$ be a quasi-multiplier on $K_{A}$ and let $w, x, y \in K_{A}$ and $\alpha \in \mathbb{C}$. By Theorem 5.2 there exist elements $z \in K_{A}^{+}$and $u, v \in K_{A}$ such that $x=u z$ and $y=v z$. Then

$$
\begin{aligned}
m(\alpha x+y, w) & =m((\alpha u+v) z, w)=(\alpha u+v) m(z, w) \\
& =\alpha m(u z, w)+m(v z, w)=\alpha m(x, w)+m(y, w) .
\end{aligned}
$$

Similarly we can prove that $m(w, \alpha x+y)=\alpha m(w, x)+m(w, y)$; that is, $m$ is bilinear.

Let $\delta\left(K_{A}\right)$ denote the space of all quasi-multipliers on $K_{A}$ The members of $\delta\left(K_{A}\right)$ are not, in general, continuous However, for any $m \operatorname{tn} \delta(K$, it is 
easy to show that $a \circ m \circ b \in Q M\left(K_{A}\right)$ for $a, b \in K_{A}$, and that $\|a \circ m \circ b\| \leq$ $\|m(a, b)\|$. Thus we can define the quasi-strict topology $\gamma$ on $\delta\left(K_{A}\right)$; that is, $\gamma$ is determined by the family of semi-norms $\left\{\gamma_{a, b}: a, b \in K_{A}\right\}$, where $\gamma_{a, b}(m)=\|a \circ m \circ b\|\left(m \in \delta\left(K_{A}\right)\right)$. Since every $C^{*}$-algebra has a bounded a.i., it follows that $K_{A}$ has a bounded a.i. Suppose that $\left\{e_{\alpha}: \alpha \in I\right\}$ is a bounded a.i. for $K_{A}$, with $\left\|e_{\alpha}\right\| \leq C$ for all $\alpha \in I$. Then, for $m \in \delta\left(K_{A}\right), a, b \in K_{A}$,

$$
\begin{aligned}
\|a \circ m \circ b\| & =\|\Phi(m(a, b))\| \geq \limsup _{\alpha}\left\|\Phi(m(a, b))\left(\frac{e_{\alpha}}{C}, \frac{e_{\alpha}}{C}\right)\right\| \\
& =\frac{1}{C^{2}} \lim _{\alpha}\left\|e_{\alpha} m(a, b) e_{\alpha}\right\|=\frac{1}{C^{2}}\|m(a, b)\| .
\end{aligned}
$$

Thus

$$
C^{2}\|a \circ m \circ b\| \geq\|m(a, b)\| \geq\|a \circ m \circ b\|,
$$

which implies that the $\gamma$-topology on $\delta\left(K_{A}\right)$ may also be defined by the semi-norms $m \rightarrow\|m(a, b)\|$. In the sequel we find it more convenient to work with this family of semi-norms to establish properties of the locally convex space $\left(\delta\left(K_{A}\right), \gamma\right)$.

THEOREM 5.3. $\delta\left(K_{A}\right)$ is $\gamma$-complete.

Proof. Let $\left\{m_{\alpha}\right\}$ be a $\gamma$-Cauchy net in $\delta\left(K_{A}\right)$. Then, for $a, b \in K_{A}$, $\left\{m_{\alpha}(a, b)\right\}$ is a Cauchy net in $K_{A}$ and so $\lim _{\alpha} m_{\alpha}(a, b)$ exists in $A$. Define $m(a, b)=\lim _{\alpha} m_{\alpha}(a, b)$. It is clear that, for all $c, d \in K_{A}, m(c a, b d)=$ $\mathrm{cm}(a, b) d$. By Theorem 5.2 there exist elements $u, v, w, w^{\prime}$ in $K_{A}$ such that $a=w u$ and $b=v w^{\prime}$, and so

$$
m(a, b)=\lim _{\alpha} w m_{\alpha}(u, v) w^{\prime} \in K_{A} .
$$

Thus $m \in \delta\left(K_{A}\right)$, and since $\gamma$ - $\lim _{\alpha} m_{\alpha}=m$ it follows that $\delta\left(K_{A}\right)$ is $\gamma$-complete.

The Pedersen ideal is a $*$-ideal ([17], Lemma 1.1), and so the equation

$$
m^{*}(a, b)=\left(m\left(b^{*}, a^{*}\right)\right)^{*}
$$

defines an element of $\delta\left(K_{A}\right)$. The mapping $m \rightarrow m^{*}$ defines an involution on $\delta\left(K_{A}\right)$ and is continuous with respect to the $\gamma$-topology; for, if $m=\gamma-$ $\lim _{\alpha} m_{\alpha}$, then, for any $x, y \in K_{A}$,

$$
\begin{aligned}
\lim _{\alpha} m_{\alpha}^{*}(x, y) & =\lim _{\alpha}\left(m_{\alpha}\left(y^{*}, x^{*}\right)\right)^{*}=\left(\lim _{\alpha} m_{\alpha}\left(y^{*}, x^{*}\right)\right)^{*} \\
& =\left(m\left(y^{*}, x^{*}\right)\right)^{*}=m^{*}(x, y),
\end{aligned}
$$

which implies that $m^{*}=\gamma$ - $\lim _{\alpha} m_{\alpha}^{*}$.

For the next two results we assume that $K_{A}$ contains a bounded central a.i. This is the case if, for example, $A$ is a quasi-central $C^{*}$-algebra. The notion of a quasi-central $C^{*}$-algebra was considered by Archbold in [2], where a $C^{*}$-algebra is defined to be quasi-central if no primitive ideal contains the centre. A $C^{*}$-algebra is quasi-central if and only if it has a bounded a.i. which helongs to the centre $Z(A)$ of $A$ ([2], Proposition 1), and Archbold also proved ([2], Theorem 3) that, if $I$ is an ideal of $A$, then $\overline{I \cap Z(A)}=\bar{I} \cap Z(A)$. Thus, in the special case when $l=K_{A}, K_{A} \cap Z(A)$ is dense in $Z(A)$, and so, if $Z(A)$ contains a bounded a.i., then $K_{A}$ contains a bounded a.i. which is central.

THEORHM 5.4. Let $A$ be a $C^{*}$-algebra and suppose that $K_{A}$ contains a bounded central a.t. Then $K_{A}$ is $\gamma$-dense in $\delta\left(K_{A}\right)$.

Prool. Lot $\left\{f_{n}\right\}$ be an a.i. in $K_{A}$ with the required property and suppose that, $m \in \delta\left(K_{A}\right)$. Then, for any $a, b \in K_{A}$,

$$
\begin{aligned}
m(a, b) & =\lim _{\alpha} e_{\alpha x} m(a, b) e_{\alpha}=\lim _{\alpha} m\left(e_{\alpha} a, b e_{\alpha}\right) \\
& =\lim _{\alpha} a m\left(e_{\alpha}, e_{\alpha}\right) b=\lim _{\alpha} \Phi\left(m\left(e_{\alpha}, e_{\alpha}\right)\right)(a, b),
\end{aligned}
$$

which implies that $m=\gamma-\lim _{\alpha} \Phi\left(m\left(e_{\alpha}, e_{\alpha}\right)\right)$; that is, $K_{A}$ is $\gamma$-dense in $\delta\left(K_{A}\right)$

THeOrmM 5.5. Let $A$ and $B$ be $C^{*}$-algebras and suppose that $K_{A}$ has a bounded central a.i. If $\phi$ is a *-homomorphism of $A$ onto $B$, then and

(i) $\phi$ can be extended to a *-linear mapping $\tilde{\phi}$ of $\delta\left(K_{A}\right)$ into $\delta\left(K_{B}\right)$,

(ii) the mapping $\widetilde{\phi}$ is $\gamma$-continuous.

Proof. We first note that $\phi$ is norm decreasing ([18], p. 16, Theorem 1.5.7) and that $\phi\left(K_{A}\right)=K_{B}\left([17]\right.$, Corollary 6). Let $b_{1}, b_{2} \in K_{B}$ and suppose that $x_{1}, x_{2}, y_{1}, y_{2}$ are elements of $K_{A}$ such that $\phi\left(x_{1}\right)=\phi\left(y_{1}\right)=b_{1}$ and $\phi\left(x_{2}\right)=\phi\left(y_{2}\right)=b_{2}$. If $\left\{e_{\alpha}\right\}$ is a central bounded a.i. in $K_{A}$, then, for any $m \in \delta\left(K_{A}\right)$

$$
\begin{aligned}
\phi\left(m\left(x_{1}, x_{2}\right)\right) & =\lim _{\alpha} \phi\left(e_{\alpha} m\left(x_{1}, x_{2}\right) e_{\alpha x}\right)=\lim _{\alpha} \phi\left(m\left(x_{1} e_{\alpha}, e_{\alpha} x_{2}\right)\right) \\
& =\lim _{\alpha} \phi\left(x_{1}\right) \phi\left(m\left(e_{\alpha}, e_{\alpha}\right)\right) \phi\left(x_{2}\right) \\
& =\lim _{\alpha} \phi\left(y_{1}\right) \phi\left(m\left(e_{\alpha x}, e_{\alpha}\right)\right) \phi\left(y_{2}\right)=\phi\left(m\left(y_{1}, y_{2}\right)\right) .
\end{aligned}
$$

Thus the equation $\widetilde{\phi}(m)\left(b_{1}, b_{2}\right)=\phi\left(m\left(x_{1}, x_{2}\right)\right)$ defines a mapping $\widetilde{\phi}(m)$ : $K_{B} \times K_{B} \rightarrow K_{B}$. It is routine to show that $\tilde{\phi}(m) \in \delta\left(K_{B}\right)$ and that the mapping $m \rightarrow \widetilde{\phi}(m)$ of $\delta\left(K_{A}\right)$ into $\delta\left(K_{B}\right)$ is linear and is an extension of $\phi$. To complete the proof of (i) we show that $m^{*} \rightarrow(\tilde{\phi}(m))^{*}$. Let $a, b \in K_{B}$ and suppose that $a=\phi(x), b=\phi(y)$, where $x$ and $y$ are in $K_{A}$. Then, since 
$\phi$ is a $*$-homomorphism, $a^{*}=\phi\left(x^{*}\right)$ and $b^{*}=\phi\left(y^{*}\right)$. Thus

$$
\begin{aligned}
\left(\tilde{\phi}\left(m^{*}\right)\right)(a, b) & =\phi\left(m^{*}(x, y)\right)=\phi\left(\left(m\left(y^{*}, x^{*}\right)\right)^{*}\right)=\left(\phi\left(m\left(y^{*}, x^{*}\right)\right)\right)^{*} \\
& =\left((\tilde{\phi}(m))\left(b^{*}, a^{*}\right)\right)^{*}=(\tilde{\phi}(m))^{*}(a, b)
\end{aligned}
$$

that is, $\widetilde{\phi}\left(m^{*}\right)=(\widetilde{\phi}(m))^{*}$, as required.

To prove (ii), let $b_{1}, b_{2}$ be any elements of $K_{B}$ and suppose that $x_{1}, x_{2} \in$ $K_{A}$ are such that $\phi\left(x_{1}\right)=b_{1}$ and $\phi\left(x_{2}\right)=b_{2}$. Then

$$
\left\|\widetilde{\phi}(m)\left(b_{1}, b_{2}\right)\right\|=\left\|\phi\left(m\left(x_{1}, x_{2}\right)\right)\right\| \leq\left\|m\left(x_{1}, x_{2}\right)\right\|,
$$

which implies that $\tilde{\phi}:\left(\delta\left(K_{A}\right), \gamma\right) \rightarrow\left(\delta\left(K_{B}\right), \gamma\right)$ is contiuuous.

LEMMA 5.6. The sets

$$
V_{a, b}=\left\{m \in \delta\left(K_{A}\right):\|m(a, b)\| \leq 1, a, b \in K_{A}^{+}\right\}
$$

form a neighbourhood base at 0 for the $\gamma$-topology on $\delta\left(K_{A}\right)$.

Proof. Clearly $V_{a, b}$ is a $\gamma$-neighbourhood of 0 in $\delta\left(K_{A}\right)$ for each $a, b \in$ $K_{A}^{+}$. On the other hand, let $U$ be any $\gamma$-neighbourhood of 0 . Then there exist elements $x_{1}, \ldots, x_{n}, y_{1}, \ldots, y_{m}$ in $K_{A}$ such that

$$
\left\{m \in \delta\left(K_{A}\right):\left\|m\left(x_{i}, y_{j}\right)\right\| \leq 1(i=1, \ldots, n, j=1, \ldots, m)\right\} \subseteq U .
$$

By Theorem 5.2 there exist $a_{1}, b_{1} \in K_{A}^{+}$and $u_{1}, \ldots, u_{n_{k}}, v_{1}, \ldots, v_{m}$ in $K_{A}$ such that

$$
x_{i}=u_{i} a_{1}(i=1, \ldots, n) \text { and } y_{j}=b_{1} v_{j}(j=1, \ldots, m) .
$$

Let $M=\max \left\{\left\|u_{i}\right\|\left\|v_{j}\right\|: 1 \leq i \leq n, 1 \leq j \leq m\right\}$ and let $a=\sqrt{M} a_{1}, b=$ $\sqrt{M} b_{1}$. Then $a, b \in K_{A}^{+}$and, for any $m \in V_{a, b}, i=1, \ldots, n, j=1, \ldots, m$,

$$
\begin{aligned}
\left\|m\left(x_{i}, y_{j}\right)\right\| & =\left\|m\left(u_{i} a_{1}, b_{1} v_{j}\right)\right\| \leq\left\|u_{i}\right\|\left\|m\left(a_{1}, b_{1}\right)\right\|\left\|v_{j}\right\| \\
& \leq\|m(a, b)\| \leq 1
\end{aligned}
$$

which implies that $V_{a, b} \subseteq U$, as required.

We now establish a characterization for the $\gamma$-dual of $\delta\left(K_{A}\right)$.

Theorem 5.7. Let $A$ be a $C^{*}$-algebra and $K_{A}$ its Pedersen ideal. Then

$$
\left(\delta\left(K_{A}\right), \gamma\right)^{*}=\left\{a \cdot g \cdot b: a, b \in K_{A}^{*}, g \in A^{*}\right\},
$$

where $a \cdot g \cdot b$ is the functional on $\delta\left(K_{A}\right)$ defined by $(a \cdot g \cdot b)(m)=g(m(b, a))$.

Proof. We note that when $a \cdot g \cdot b$ is restricted to $A$ it agrees with the usual Arens product of $a, b$ and $g$; this justifies our use of the notation to define the functional $a \cdot g \cdot b$. Since

$$
|(a \cdot g \cdot b)(m)| \leq\|g\|\|m(b, a)\| \quad\left(m \in \delta\left(K_{A}\right)\right)
$$

it follows that $a \cdot g \cdot b \in\left(\delta\left(K_{A}\right), \gamma\right)^{*}$ for each $a, b \in K_{A}^{+}$and $g \in A^{*}$.
On the other hand, suppose that $f \in\left(\delta\left(K_{A}\right), \gamma\right)^{*}$. By Lemma 5.6 there exist $a, b \in K_{A}^{+}$such that $|f(m)| \leq 1$ whenever $\|m(b, a)\| \leq 1$. This implies that, for any $m \in \delta\left(K_{A}\right),|f(m)| \leq\|m(b, a)\|$. On the subspace $W_{a, b}=$ $\left\{m(b, a): m \in \delta\left(K_{A}\right)\right\}$ we define the functional $g$ by

$$
g(m(b, a))=f(m) \text {. }
$$

It is clear that $g$ is well defined and that $g \in\left(W_{a, b},\|\cdot\|\right)^{*}$, with $\|g\| \leq 1$. By the Haln Bandach theorem $g$ has a continuous extension to all of $A$; we retain the notation $y$ to denote the extension so that $g \in A^{*}$ and $\|g\| \leq 1$. By the first part of the proof $a \cdot g \cdot b \in\left(\delta\left(K_{A}\right), \gamma\right)^{\prime}$. Moreover, for any $m \in \delta\left(K_{A}\right)$, $(a \cdot g \cdot b)(m)=g(m(b, a))=f(m)$; that is, $f=a \cdot g \cdot b$.

Let $\left\{A_{i}: i \in I\right\}$ be a family of $C^{*}$-algebras and let $A=\left(\sum_{i \in I} A_{i}\right)_{0}$; that is, $A$ consists of those elements $a=\left(a_{i}\right)$ such that, for each $\varepsilon>0$, the set $\left\{i \in I:\left\|a_{i}\right\| \geq \varepsilon\right\}$ is finite. With the usual operations of addition and multiplication, $A$ is a $C^{*}$-algebra, the norm being given by $\|a\|=\sup _{i}\left\|a_{i}\right\|$ and the involution by $a^{*}=\left(a_{i}^{*}\right)$. If $K_{A_{i}}$ denotes the Pedersen ideal of $A_{i}$, then the Pedersen ideal $K_{A}$ of $A$ consists of those elements $\left(a_{i}\right)$ such that $a_{i} \in K_{A_{i}}$ and $a_{i}=0$ except for a finite number of the $i$ 's. We let $\gamma$ and $\gamma_{i}$ denote the quasi-strict topologies on $\delta\left(K_{A}\right)$ and $\delta\left(K_{A_{i}}\right)$ respectively.

THEORMM 5.8. Let $\left\{A_{i}: i \in I\right\}$ be a family of $C^{*}$-algebras and suppose that each $A_{i}$ has a minimal central a.i. If $A=\left(\sum_{i \in I} A_{i}\right)_{0}$, then $\delta\left(K_{A}\right)$ is topologically *-isomorphic to $\prod_{i \in I} \delta\left(K_{A_{i}}\right)$, the topology on $\delta\left(K_{A}\right)$ being $\gamma$ and that of $\prod_{i \in I} \delta\left(K_{A_{i}}^{\prime}\right)$ being the product of the spaces $\left(\delta\left(K_{A_{i}}\right), \gamma_{i}\right)$.

Proof. For each $i \in I, \phi_{i}(a)=a_{i}\left(a=\left(a_{i}\right) \in A\right)$ defines a natural *-homomorphism $\phi_{i}$ of $A$ onto $A_{i}$. The hypothesis ensures that $A$ has a bounded central a.i. and so by Theorem 5.5 each $\phi_{i}$ has an extension to a *-linear mepping, $\widetilde{\phi}_{i}$ say, of $\delta\left(K_{A}\right)$ into $\delta\left(K_{A_{i}}\right)$. Each $\widetilde{\phi}_{i}$ is given by the equation

$$
\left(\tilde{\phi}_{i}(m)\right)\left(a_{i}, b_{i}\right)=\phi_{i}\left(m\left(\widetilde{a}_{i}, \widetilde{b}_{i}\right)\right) \quad\left(m \in \delta\left(K_{A}\right)\right),
$$

where $a_{i} \rightarrow \tilde{a}_{i}$ is the uatural embedding of $K_{A_{i}}$ into $K_{A_{A}}$.

Wo define a mapping $\xi: \delta\left(K_{A}\right) \rightarrow \prod_{i \in I} \delta\left(K_{A_{i}}\right)$ by

$$
\xi(m)=\left(\tilde{\phi}_{i}(m)\right) \text {. }
$$

Clearly $\xi$ is a *ulinear mapping. It is also surjective, as follows. Let $\left(m_{i}\right)$ be any element of $\prod_{i \in I} \delta\left(K_{A_{i}}\right)$. If $a, b$ are any elements of $K_{A}$, then $a_{i}=0$ and $b_{j}=0$ except for a finite number of $i$ 's and $j$ 's and so $m_{i}\left(a_{i}, b_{i}\right)=0$ except for a finite number of $i$ 's. Thus the equation

$$
(m(a, b))_{i}=m_{i}\left(a_{i}, b_{i}\right) \quad(i \in I)
$$

defines a mapping $m: K_{A} \times K_{A} \rightarrow K_{A}$ and it is easy to see that $m \in \delta\left(K_{A}\right)$. 
Moreover, $\xi(m)=\left(m_{i}\right)$; for, if $j$ is any index in $I$, then

$$
\left(\tilde{\phi}_{j}(m)\right)\left(a_{j}, b_{j}\right)=\phi_{j}\left(m\left(\widetilde{a}_{j}, \tilde{b}_{j}\right)\right)=\left(m\left(\widetilde{a}_{j}, \widetilde{b}_{j}\right)\right)_{j}=m_{j}\left(a_{j}, b_{j}\right),
$$

which implies that $\tilde{\phi}_{j}(m)=m_{j}$; that is, $\xi(m)=\left(m_{i}\right)$, as required.

It is clear that $\xi$ is injective. Finaliy, we show that $\xi$ is topological. Let

$U$ be any neighbourhood of 0 in $\prod_{i \in I} \delta\left(K_{A_{i}}\right)$. Then there exist clements $a_{i_{j}}, b_{i_{j}} \in K_{A_{i j}}(1 \leq j \leq n)$ such that

$$
\left\{\left(m_{i}\right):\left\|m_{i_{j}}\left(a_{i_{j}}, b_{i_{j}}\right)\right\| \leq 1,1 \leq j \leq n\right\} \subseteq U .
$$

Let $a=\sum_{j=1}^{n} \tilde{a}_{i_{j}}, b=\sum_{j=1}^{n} \tilde{b}_{i_{j}}$ and $V=\left\{m \in \delta\left(K_{A}\right):\|m(a, b)\| \leq 1\right\}$. If $m \in V$, then, for $1 \leq j \leq n$,

$$
\left\|\left(\tilde{\phi}_{i_{j}}(m)\right)\left(a_{i_{j}}, b_{i_{j}}\right)\right\|=\left\|\phi_{i_{j}}\left(m\left(\widetilde{a}_{i_{j}}, \tilde{b}_{i_{j}}\right)\right)\right\| .
$$

Since $\phi_{i_{j}}\left(\widetilde{a}_{i_{j}}\right)=\phi_{i_{j}}(a)$ and $\phi_{i_{j}}\left(\tilde{b}_{i_{j}}\right)=\phi_{i_{j}}(b)$, it follows from the proof of Theorem 5.5 that

$$
\left\|\phi_{i_{j}}\left(m\left(\widetilde{a}_{i_{j}}, \widetilde{b}_{i_{j}}\right)\right)\right\|=\left\|\phi_{i_{j}}(m(a, b))\right\| .
$$

Thus

$$
\left\|\left(\widetilde{\phi}_{i_{j}}(m)\right)\left(a_{i_{j}}, b_{i_{j}}\right)\right\|=\left\|(m(a, b))_{i_{j}}\right\|=\left\|m_{i_{j}}\left(a_{i_{j}}, b_{i_{j}}\right)\right\| \leq 1
$$

that is, $\xi(V) \subseteq U$, and so $\xi$ is continuous.

On the other hand, suppose that $a, b \in K_{A}$. Then $a_{i}=0$ (resp. $b_{j}=0$ ) except for $i$ (resp. $j$ ) in a finite set of indices, say $I_{1}$ (resp. $I_{2}$ ). Suppose that $\left(m_{i}\right)$ satisfies $\left\|m_{i}\left(a_{i}, b_{i}\right)\right\| \leq 1$ for $i \in I_{1} \cup I_{2}$. Then

$$
\left\|\xi^{-1}\left(m_{i}\right)(a, b)\right\|=\sup _{i}\left\|m_{i}\left(a_{i}, b_{i}\right)\right\| \leq 1
$$

which implies that $\xi^{-1}$ is continuous, as required.

The authors would like to thank the referee for several helpful suggestions.

\section{References}

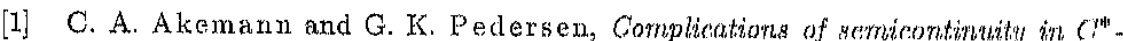
algebra theory, Duke Math. J. 40 (1973), 785-795.

[2] R. J. Archbold, Density theorems for the centre of a $C^{4}$-algebra, J. Icouclon Muth. Soc. (2) $10(1975), 1.89-197$.

[3] F. F. Bonsall and J. Duncan, Complete Normed Algebras, Springer, 1973.

[4] J. Cigler, V. Losert and P. Michnor, Banach Modules and Funnotors on Categories of Banach Spaces, Lecture Notes in Pure and Appl. Math. 46, Dekker, 1979

[5] J. Diestel and J. J. Uhl, Jr., Vector Measures, Math. Surveys 15, Amer. Math. Soc., 1977

[6] R. S. Doran and J. Wichmann, Approximate Identities and Factorization in Banach Modules, Lecture Notes in Math. 768, Springer, 1979.
7] L. Gillun an and M. Jerison, Rings of Continuous Functions, Van Nostrand, 1960.

8] M. Grosser, Module tensor products of $K_{0}(X, X)$ with its dual, in: Functions, Series, Oporators, Budapest, 1980, Colloq. Math. Soc. János Bolyai 35, NorthHollanid, 1983, 551-560.

[9] --- Arens semi-regularity of the algebra of compact operators, Illinois J. Math. 31 (1987), 544 573 .

[10] B. E. Johnsoln, An introduetion to the theory of centralizers, Proc. London Math. Soc. (3) $14(1964), 299+320$.

[11] M.S. Kussem and K. Rowlands, The quasi-strict topology on the space of quasimiultipliers of a $\mathrm{B}^{*}$-algebra, Math. Proc. Cambridge Philos. Soc. 101 (1987), 5555666.

[12] -..., , , Double multiphliers and $A^{*}$-algebras of the first kind, ibid. 102 (1987), 507516.

[13] H. Xin, The: strueture of quasi-multipliers of $C^{*}$-algebras, Trans. Amer. Math. Soc. $315(1987), 147-172$.

[14] K. MeKennon, Quilsi-multipliers, ibid. 233 (1977), 105-123.

[15] G. J. Murphy, $C^{*}$-algebras and Operator Theory, Academic Press, 1990.

[16] G. K. Pedersen, Measure theory for $C^{*}$-algebras, Math Scand 19 (1966), 131-145. -... A decomposition theory for $C^{*}$-algebras, ibid. 22 (1968), 266-268.

18] --., C*-algebros and their Automorphism Groups, Academic Press, 1979

[19] C. E. Rickart, General Theory of Banach Algebras, Van Nostrand, 1960

20] B. J. Tomink, Multipliers on Banach algebras, Studia Math. 54 (1976), 267-283.

21] R. Vastidevan and S. Goel, Embedding of quasi-multipliers of a Banach algebra into its secrond dual, Math. Proc. Cambridge Philos. Soc. 95 (1984), 457-466.

[22] ..., ,..., Quasimmultipliers and normed full direct sum of Banach algebras, Ann. Soc. Sci. Bruxelles $99(1985), 85 \cdots 95$.

[23] Y.-C. Wong, Schwurtz Spaces, Nuclear Spaces and Tensor Products, Lecture Notes in Math. 726, Springer, 1979.

DFPATTMTNT OF MATTEMATTCS

FACUI'TYY OF EDUCA'TION

DEPARTMENT OF MATHEMATICS

GAZI UNIYERSTITY

HE UNIVERSTTY COLLEGE OF WALES

'TEKNIK-OKULLAT

ABERYSTWYTH DYFED SY23 3BZ, U.K. 